\title{
Motivic integration on the Hitchin fibration
}

\author{
François Loeser and Dimitri Wyss
}

\begin{abstract}
We prove that the moduli spaces of twisted $\mathrm{SL}_{n^{-}}$and $\mathrm{PGL}_{n}$-Higgs bundles on a smooth projective curve have the same (stringy) class in the Grothendieck ring of rational Chow motives. On the level of Hodge numbers, this was conjectured by Hausel and Thaddeus, and recently proven by Groechenig, Ziegler and the second author. To adapt their argument, which relies on $p$-adic integration, we use a version of motivic integration with values in rational Chow motives and the geometry of Néron models to evaluate such integrals on Hitchin fibres.
\end{abstract}

\section{Introduction}

The main goal of the present work is to provide a proof of a motivic version of the topological mirror symmetry conjecture of Hausel and Thaddeus [HT03].

\subsection{Background on the topological mirror symmetry conjecture}

Let $C$ be a connected, smooth and projective complex curve, and let $G$ be a Lie group. A $G$-Higgs bundle on $C$ is a pair $(E, \theta)$ with $E$ a principal $G$-bundle on $C$ and $\theta$ an element of $H^{0}\left(C\right.$, ad $\left.E \otimes K_{C}\right)$, with ad $E=E \otimes_{G} \mathfrak{g}$ the adjoint vector bundle of $G$ and $K_{C}$ the canonical bundle of $C$. By assigning to $(E, \theta)$ the characteristic polynomial of $\theta$, one defines the Hitchin fibration on the corresponding moduli space. Hausel and Thaddeus conjectured in [HT03] that the moduli spaces of $\mathrm{SL}_{n^{-}}$and $\mathrm{PGL}_{n}$-Higgs bundles are mirror partners in the sense of Strominger-YauZaslow. In this case, the two Hitchin fibrations have the same base, and Hausel and Thaddeus proved that over a dense open set, their fibres are torsors under dual abelian varieties. They also conjectured that the two moduli spaces have the same Hodge numbers when defined in an appropriate way.

More precisely, fix a line bundle $L$ of degree $d$ on $C$ with $d$ prime to $n$. We consider the moduli space $\mathrm{M}_{n}^{L}$ of semi-stable $L$-twisted $\mathrm{SL}_{n}$-Higgs bundles on $C$, which is a smooth quasi-projective variety. One can also consider the moduli space $\widehat{\mathrm{M}}_{n}^{d}$ of semi-stable $\mathrm{PGL}_{n}$-Higgs bundles of degree $d$ on $C$. It is an orbifold which can be identified with the geometric quotient of $\mathrm{M}_{n}^{L}$ by the natural action of $\Gamma=\operatorname{Pic}(C)[n]$ given by twisting the underlying vector bundle of a Higgs field.

The topological mirror symmetry conjecture of Hausel and Thaddeus [HT03], now proven by

Received 6 January 2020, accepted in final form 2 July 2020.

2020 Mathematics Subject Classification 14H60, $14 \mathrm{E} 18$.

Keywords: Hitchin fibration, motivic integration.

This journal is (C) Foundation Compositio Mathematica 2021. This article is distributed with Open Access under the terms of the Creative Commons Attribution Non-Commercial License, which permits non-commercial reuse, distribution, and reproduction in any medium, provided that the original work is properly cited. For commercial re-use, please contact the Foundation Compositio Mathematica.

The authors were partially supported by the ANR grant ANR-15-CE40-0008 (Défigéo). F.L. was also supported by the Institut Universitaire de France and D.W. by the Fondation Sciences Mathématiques de Paris, under ANR-10-LABX0098. 


\section{Motivic Integration ON THE Hitchin FibRATion}

Groechenig, Ziegler and the second author in [GWZ20], is the following statement (see Theorem 5.3.1 for a slightly more general statement).

Theorem 1.1.1. Let $d$ be an integer prime to $n$ and $L$ a line bundle on $C$ of degree $d$. Then there is an equality of Hodge numbers

$$
h^{p, q}\left(\mathrm{M}_{n}^{L}\right)=h_{\mathrm{st}}^{p, q}\left(\widehat{\mathrm{M}}_{n}^{d}, \alpha_{L}^{d}\right) .
$$

A few words are in order to explain the meaning of the right-hand side of this equality. Stringy Hodge numbers are invariants introduced by Batyrev [Bat98, Bat99] for algebraic varieties with log terminal singularities. They are especially useful to extend topological mirror symmetry for pairs of singular Calabi-Yau varieties beyond the smooth case. The numbers appearing on the right-hand side are twisted versions of the stringy Hodge numbers of $\widehat{\mathrm{M}}_{n}^{d}$. The twisting involves a $\mu_{n}$-gerbe $\alpha_{L}$ on $\widehat{\mathrm{M}}_{n}^{d}$ that, roughly speaking, describes the duality between generic Hitchin fibres, which are torsors under dual abelian varieties. Concretely, $\alpha_{L}$ has the effect of singling out certain isotypical components, as we explain next.

\subsection{Hodge-Deligne polynomials}

In this subsection, we assume $k=\mathbb{C}$. Let $M$ be a smooth connected variety over $k$ with an action of a finite abelian group $\Gamma$ preserving its canonical bundle. For $\gamma \in \Gamma$ and a point $x$ in $M$ fixed by $\gamma$, one defines an integer $w_{x}(\gamma)$ in terms of the eigenvalues of $\gamma$ acting on the tangent space at $x$; cf. Definition 3.1.5. In the cases we will consider, $w_{x}(\gamma)$ is constant on the fixed point set $M^{\gamma}$ for each $\gamma \in \Gamma$. Under this assumption, the stringy $E$-polynomial of the quotient $M / \Gamma$ is given by

$$
E_{\mathrm{st}}(M / \Gamma ; x, y)=\sum_{\gamma \in \Gamma}(x y)^{\operatorname{dim} M-w(\gamma)} E\left(M^{\gamma} / \Gamma ; x, y\right),
$$

with $E$ the usual Hodge-Deligne polynomial.

We now specialize to the case when $M=\mathrm{M}_{n}^{L}$ and $\Gamma=\operatorname{Pic}^{0}(C)[n]$. Let $\varrho=\langle\cdot, \cdot\rangle: \Gamma \times \Gamma \rightarrow \mu_{n}$ be the Weil pairing on $\Gamma=\operatorname{Pic}^{0}(C)[n]$ and $\varrho_{\gamma}=\langle\gamma, \cdot\rangle: \Gamma \rightarrow \mu_{n}$ the character induced by $\gamma \in \Gamma$. For any integer $s$, we obtain a twisted stringy $E$-polynomial

$$
E_{\mathrm{st}}^{\varrho^{s}}\left(\widehat{\mathrm{M}}_{n}^{d} ; x, y\right)=\sum_{\gamma \in \Gamma}(x y)^{\operatorname{dim} \mathrm{M}_{n}^{L}-w(\gamma)} E^{\varrho_{\gamma}^{s}}\left(\mathrm{M}_{n}^{L, \gamma} ; x, y\right),
$$

with $E^{\varrho_{\gamma}^{s}}\left(\mathrm{M}_{n}^{L, \gamma} ; x, y\right)$ the $E$-polynomial of the $\varrho_{\gamma}^{s}$-isotypical component of the cohomology with compact supports of $\mathrm{M}_{n}^{L, \gamma}$. As recalled in Section 5, equality (1.1.1) can be restated as the following identity between $E$-polynomials:

$$
E\left(\mathrm{M}_{n}^{L} ; x, y\right)=E_{\mathrm{st}}^{\varrho^{-1}}\left(\widehat{\mathrm{M}}_{n}^{d} ; x, y\right) .
$$

\subsection{The main result}

Let $r$ be the order of $\operatorname{Pic}(C)[n]$, and set $\Lambda=\mathbb{Q}\left(\mu_{r}\right)$. We denote by $\mathrm{M}_{\text {rat }}(k, \Lambda)$ the category of Chow motives over $k$ with coefficients in $\Lambda$, and by $K_{0}\left(\mathrm{M}_{\text {rat }}(k, \Lambda)\right)$ its Grothendieck ring. We denote by $\left[\mathrm{M}_{n}^{L}\right]$ the virtual Chow motive of $\mathrm{M}_{n}^{L}$ in $K_{0}\left(\mathrm{M}_{\text {rat }}(k, \Lambda)\right)$. One may also define by a formula similar to (1.2.1) a virtual Chow motive $\left[\widehat{\mathrm{M}}_{n}^{d}\right]_{\mathrm{st}}^{\varrho^{s}} \in K_{0}\left(\mathrm{M}_{\mathrm{rat}}(k, \Lambda)\right)$.

We set

$$
K_{0}\left(\mathrm{M}_{\mathrm{rat}}(k, \Lambda)\right)_{\text {loc }}:=K_{0}\left(\mathrm{M}_{\mathrm{rat}}(k, \Lambda)\right) \otimes_{\mathbb{Z}\left[\mathbb{L}, \mathbb{L}^{-1}\right]} \mathbb{A}
$$

with $\mathbb{A}=\mathbb{Z}\left[\mathbb{L}, \mathbb{L}^{-1},\left(1 /\left(1-\mathbb{L}^{-i}\right)\right)_{i>0}\right]$, where $\mathbb{L}$ denotes the class of the Lefschetz motive, and we denote the localization morphism by $\vartheta: K_{0}\left(\mathrm{M}_{\text {rat }}(k, \Lambda)\right) \rightarrow K_{0}\left(\mathrm{M}_{\text {rat }}(k, \Lambda)\right)_{\text {loc }}$. 


\section{F. LOESER AND D. Wyss}

We can now state the main result of this paper.

Theorem 1.3.1. The equality

$$
\vartheta\left(\left[\mathrm{M}_{n}^{L}\right]\right)=\vartheta\left(\left[\widehat{\mathrm{M}}_{n}^{d^{\prime}}\right]_{\mathrm{st}}^{\varrho^{-1}}\right)
$$

holds in $K_{0}\left(\mathrm{M}_{\text {rat }}(k, \Lambda)\right)_{\text {loc }}$.

Equality (1.2.2) follows from (1.3.1) when we apply the $E$-polynomial to both sides.

\subsection{Strategy of the proof}

The strategy of the proof of (1.1.1) in [GWZ20] is very roughly the following. After spreading out, one may assume all the data to be defined over of subalgebra $R$ of finite type over $\mathbb{Z}$. Using $p$-adic Hodge theory, one reduces the proof of (1.1.1) to proving that for every ring morphism $R \rightarrow \mathbb{F}_{q}$,

$$
\# \mathrm{M}_{n}^{L}\left(\mathbb{F}_{q}\right)=\# \text { st }\left(\widehat{\mathrm{M}}_{n}^{d}, \alpha_{L}\right)\left(\mathbb{F}_{q}\right)
$$

where on the right-hand side, the stringy number of points $\#_{\text {st }}$ is defined in a way much similar to the stringy Hodge polynomial and the twisting by $\alpha_{L}$ has the effect of replacing expressions involving numbers of points by character sums. The next step is to move from an equality between numbers of points over finite fields to an equality between $p$-adic integrals. That is, for a non-archimedean local field $F$ with ring of integers $\mathcal{O}_{F}$ and residue field $\mathbb{F}_{q}$, one has to prove

$$
\int_{\mathrm{M}_{n}^{L}\left(\mathcal{O}_{F}\right)} d \mu=\int_{\widehat{\mathrm{M}}_{n}^{d}\left(\mathcal{O}_{F}\right)} f_{\alpha_{L}^{d}} d \mu_{\mathrm{orb}}
$$

Here $d \mu$ is the canonical $p$-adic measure on $\mathrm{M}_{n}^{L}\left(\mathcal{O}_{F}\right)$, the measure $d \mu_{\text {orb }}$ is the corresponding orbifold measure on $\widehat{\mathrm{M}}_{n}^{d}\left(\mathcal{O}_{F}\right)$, and $f_{\alpha_{L}^{d}}$ is a certain function assigned to $\alpha_{L}^{d}$. That this reduction is possible is due to the fact that the $p$-adic volume of an orbifold over the ring of integers of a nonarchimedean local field can be expressed in terms of stringy point-counting over the residue field. In fact, similar statements for motivic volumes can already be found in [DL02, Yas04, Yas06] in connection with the McKay correspondence. The proof of (1.4.1) proceeds by using a Fubini theorem for the two Hitchin fibrations. Outside a bad locus of measure zero in the common base of the two Hitchin fibrations, the fibres behave well; in particular, they are torsors under dual abelian varieties. By a Fubini theorem, it is thus enough to prove that for any point $a$ in the base which does not belong to the bad locus, the two fibre integrals over $a$ with respect to the relative measures are equal. This is where Tate duality for abelian varieties over non-archimedean local fields enters the game. For instance, when the fibre of $\mathrm{M}_{n}^{L}\left(\mathcal{O}_{F}\right)$ over $a$ has no rational points, in which case the corresponding fibre integral is zero, the authors of [GWZ20] are able to show, using Tate duality, that the function $f_{\alpha_{L}^{d}}$ behaves like a non-trivial character on the fibre over $\widehat{\mathrm{M}}_{n}^{d}\left(\mathcal{O}_{F}\right)$, which implies that the second fibre integral is also zero.

In the present paper, we use a variant of the theory of motivic integration developed by Cluckers and the first author in [CL08], but for functions with values in a Grothendieck rings of Chow motives with coefficients in a characteristic zero field $\Lambda$ containing enough roots of unity. It satisfies a Fubini theorem, as does the original theory, which is crucial for our needs. One of the important features of working with Chow motives is that in the presence of finite group action, Chow motives may decompose into isotypical components. They can be viewed as motivic analogues of character sums over finite fields. This analogy works quite well; for instance, there is a motivic version of the fact that non-trivial character sums on commutative algebraic groups are zero. 


\section{MotiviC INTEGRATion ON THE Hitchin FibRATion}

Our strategy for proving our main result follows the main lines of [GWZ20], replacing $p$-adic integration with motivic integration. We start by expressing the equality we want to prove as an equality between motivic integrals on moduli spaces of Higgs bundles over $k \llbracket t \rrbracket$. In order to extract twisted stringy invariants from the motivic volume of $\widehat{\mathrm{M}}_{n}^{d}$, we prove an equivariant volume formula for orbifolds, Theorem 3.1.6, which might be of independent interest. Applying the Fubini theorem to the corresponding Hitchin fibrations, one then reduces the proof of the equality to comparing fibre integrals outside a bad locus of measure zero in the common base of the Hitchin fibrations. As Tate duality is not available in our context, we then argue directly with the Weil pairing on $\Gamma$ and its interaction with Néron models of generic Hitchin fibres.

\subsection{Organization of the paper}

The paper is organized as follows. Section 2 is devoted to the development of the Chow motive variant of the theory in [CL08] and also contains some background and complements about Chow motives and motivic integration. In Section 3, we study motivic volumes of orbifolds over $k \llbracket t \rrbracket$ in this framework. We show, in particular, that these can be computed on the coarse moduli space of the inertia stack of the special fibre. This can be seen as an extension of previous work in [DL02, Yas04, Yas06]. Section 4 is devoted to Néron models and their relation to pairings arising from self-dual isogenies. Our main result, Theorem 5.3.3, is presented in Section 5. We then tie all the preliminary results together in Section 6, where we complete the proof of Theorem 5.3.3.

\section{Background and complements on motivic integration}

\section{1 Étale motives and their Grothendieck rings}

Let $k$ be a field of characteristic zero and $S$ a quasi-projective $k$-scheme. Fix a field $\Lambda$ of characteristic zero. We will work with the triangulated category $\operatorname{DA}^{\text {ét }}(S, \Lambda)$ of étale motives over $S$ with coefficients in $\Lambda$ introduced by Ayoub. It was first introduced in [Ayo07b, Définition 4.5.21] (under a different name), and its construction is recalled at the beginning of Section 3 of [Ayo14b]. By [Ayo14b, Proposition 3.2] and [Ayo07a, Scholie 1.4.2], it is endowed with a Grothendieck six operations formalism. In the paper [Ayo14b], Ayoub also introduced the full triangulated subcategory $\operatorname{DA}_{\mathrm{ct}}^{\text {ét }}(S, \Lambda)$ of constructible étale motives, for which he proved stability under the six operations in Théor̀emes 8.10 and 8.12 of [Ayo14b]. We also refer to [Ayo14a] for an accessible and useful introduction.

We denote by $K_{0}\left(\operatorname{Var}_{S}\right)$ the Grothendieck ring of varieties over $S$ and by $\mathcal{M}_{S}$ its localization by the class $\mathbb{L}$ of the affine line over $S$.

In [IS13, Lemma 2.1], Ivorra and Sebag prove the existence of a unique ring morphism

$$
\chi_{S, c}: \mathcal{M}_{S} \longrightarrow K_{0}\left(\mathrm{DA}_{\mathrm{ct}}^{\mathrm{ét}}(S, \Lambda)\right)
$$

which assigns to a quasi-projective $S$-scheme $p: X \rightarrow S$ the element $\chi_{S, c}([X]):=\left[p_{!}\left(\mathbb{1}_{X}\right)\right]$ in $K_{0}\left(\mathrm{DA}_{\mathrm{ct}}^{\text {ét }}(S, \Lambda)\right)$.

Now, if $q: S \rightarrow T$ is a morphism of quasi-projective $k$-schemes, the functors

$$
\begin{aligned}
& q ! \mathrm{DA}_{\mathrm{ct}}^{\text {ét }}(S, \Lambda) \\
& q^{*}: \mathrm{DA}_{\mathrm{ct}}^{\text {ét }}(T, \Lambda) \longrightarrow \mathrm{DA}_{\mathrm{ct}}^{\text {ét }}(T, \Lambda), \\
& \mathrm{DA}_{\mathrm{ct}}^{\text {et }}(S, \Lambda)
\end{aligned}
$$




\section{F. LOESER AND D. WYSS}

induce morphisms of Grothendieck rings

$$
\begin{aligned}
& q ! K_{0}\left(\mathrm{DA}_{\mathrm{ct}}^{\text {ét }}(S, \Lambda)\right) \longrightarrow K_{0}\left(\mathrm{DA}_{\mathrm{ct}}^{\text {ét }}(T, \Lambda)\right), \\
& q^{*}: K_{0}\left(\mathrm{DA}_{\mathrm{ct}}^{\text {ét }}(T, \Lambda)\right) \longrightarrow K_{0}\left(\mathrm{DA}_{\mathrm{ct}}^{\text {ét }}(S, \Lambda)\right) .
\end{aligned}
$$

Composition with $q$ and pullback induce, respectively, morphisms

$$
\begin{aligned}
q_{!}: \mathcal{M}_{S} & \longrightarrow \mathcal{M}_{T}, \\
q^{*}: \mathcal{M}_{T} & \longrightarrow \mathcal{M}_{S} .
\end{aligned}
$$

It follows directly from the constructions and Ayoub's six operations formalism for the category $\mathrm{DA}_{\mathrm{ct}}^{\text {ét }}(S, \Lambda)$ of constructible étale motives, as recalled above, that the diagrams

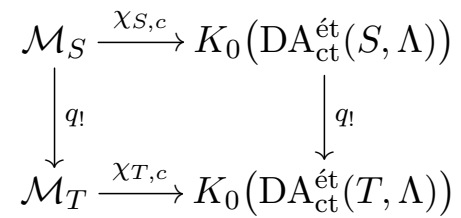

and

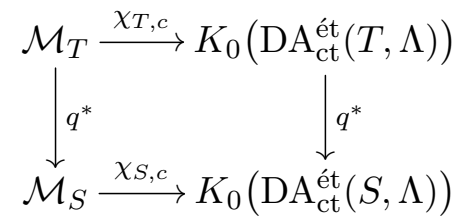

commute.

Lemma 2.1.1. Let $S$ be a quasi-projective $k$-scheme. Let $\beta \in K_{0}\left(\mathrm{DA}_{\mathrm{ct}}^{\mathrm{et}}(S, \Lambda)\right)$ be such that for every point $i_{x}: x \hookrightarrow S$, we have $i_{x}^{*}(\beta)=0$. Then $\beta=0$.

Proof. Let $i_{x}: x \hookrightarrow S$ be a point of $S$. As an $S$-scheme, it is the limit of the family of locally closed subsets $C$ of $S$ containing $x$. Since the family of locally closed subsets of the form $F \cap U$ with $F$ closed and $U$ open affine is cofinal in the family of all such subsets $C$, it follows from [Ayo14b, Corollary 3.22] that the category $\operatorname{DA}_{c t}^{\text {et }}(x, \Lambda)$ is equivalent to the 2-colimit of the categories $\mathrm{DA}_{\mathrm{ct}}^{\text {ét }}(C, \Lambda)$. In particular, the ring $K_{0}\left(\mathrm{DA}_{\mathrm{ct}}^{\text {ét }}(x, \Lambda)\right)$ is the colimit of the rings $K_{0}\left(\mathrm{DA}_{\mathrm{ct}}^{\text {ét }}(C, \Lambda)\right)$ for $C$ running over the locally closed subschemes of $S$ containing $x$. Now, assume that for every point $i_{x}: x \hookrightarrow S$, we have $i_{x}^{*}(\beta)=0$. It follows that there exists a cover of $S$ by locally closed subschemes $C$ such that $i_{C}^{*}(\beta)=0$ for every $C$. We may assume that this cover is finite and that the locally closed subschemes $C$ form a partition of $S$. One concludes by induction on the cardinality of the cover, by using that if $j: U \rightarrow S$ is an open immersion and $i: Z \rightarrow S$ is the inclusion of the complementary closed subscheme, then $\beta=j_{!} j^{*}(\beta)+i_{!} i^{*}(\beta)$.

Remark 2.1.2. When $S=$ Spec $k$, we shall write $\mathrm{DA}_{\mathrm{ct}}^{\text {ét }}(k, \Lambda), \mathcal{M}_{k}$, etc., for the categories $\mathrm{DA}_{\mathrm{ct}}^{\text {et }}(\operatorname{Spec} k, \Lambda), \mathcal{M}_{\text {Spec } k}$, etc. The additive category $\mathrm{M}_{\mathrm{rat}}(k, \Lambda)$ of Chow motives over $k$ with coefficients in $\Lambda$ embeds in $\operatorname{DA}_{\mathrm{ct}}^{\text {et }}(k, \Lambda)$ (cf. [IS13, $\left.\S 2.2 .5\right]$ ), so we have a natural ring morphism $\iota: K_{0}\left(\mathrm{M}_{\text {rat }}(k, \Lambda)\right) \rightarrow K_{0}\left(\mathrm{DA}_{\mathrm{ct}}^{\text {ét }}(k, \Lambda)\right)$. This morphism is an isomorphism since the category $\left.\mathrm{DA}_{\mathrm{ct}}^{\text {ét }}(k, \Lambda)\right)$ is equivalent to Voevodsky's category $\mathrm{DM}_{\mathrm{gm}}(k, \Lambda)$ by [Ayo14b, Appendice B] and the ring $K_{0}\left(\mathrm{DM}_{\mathrm{gm}}(k, \Lambda)\right)$ is isomorphic to $K_{0}\left(\mathrm{M}_{\mathrm{rat}}(k, \Lambda)\right)$, a statement proved by Bondarko in [Bon09] as a consequence of his theory of weight structures (in [Bon09], only the case of $\Lambda=\mathbb{Q}$ is considered, but the proofs carry over for general $\Lambda$ ). One can check that $\chi_{k, c}: \mathcal{M}_{k} \rightarrow K_{0}\left(\mathrm{DA}_{\mathrm{ct}}^{\text {ét }}(k, \Lambda)\right)$ is equal to $\iota \circ \chi_{c}$ with $\chi_{c}: \mathcal{M}_{k} \rightarrow K_{0}\left(\mathrm{M}_{\mathrm{rat}}(k, \Lambda)\right)$ the morphism considered in [DL98] (when $\Lambda=\mathbb{Q})$. 


\section{Motivic integration On the Hitchin FiBRAtion}

\subsection{Constructible motivic functions}

We shall use in this paper the formalism of constructible motivic functions developed by Cluckers and the first author in the paper [CL08]. The introduction to the paper [CHL11] may also be useful to some readers. Let us review some of the features we will use in this paper.

2.2.1 The basic framework. We fix a field $k$ of characteristic zero, and we work in the DenefPas language $\mathcal{L}_{\mathrm{DP}, k}$. It is a 3 -sorted language in the sense of first-order logic, the sorts being the valued field sort, the residue field sort and the value group sort. The language consists of the disjoint union of the language of rings with coefficients in $k((t))$ restricted to the valued field sort, of the language of rings with coefficients in $k$ restricted to the residue field sort and of the language of ordered groups restricted to the value group sort, together with two additional symbols of unary functions ac and ord from the valued field sort to the residue field and valued groups sort, respectively. Furthermore, for the value group sort, we add symbols symbols $\equiv_{n}$ for $n>1$ in $\mathbb{N}$.

A typical example of an $\mathcal{L}_{\mathrm{DP}, k}$-structure is provided by $(k((t)), k, \mathbb{Z})$ with ac interpreted as the function ac: $k((t)) \rightarrow k$ assigning to a series its first non-zero coefficient if not zero, and zero otherwise, ord interpreted as the valuation function ord: $k((t))) \backslash\{0\} \rightarrow \mathbb{Z}$ and $\equiv_{n}$ interpreted as the equivalence relation modulo $n$. More generally, for any field $K$ containing $k$, the triple $(K((t)), K, \mathbb{Z})$ is naturally an $\mathcal{L}_{\mathrm{DP}, k}$-structure.

The constructions of the paper [CL08] take place in a category $\operatorname{Def}_{k}$, also written $\operatorname{Def}_{k}\left(\mathcal{L}_{\mathrm{DP}}\right)$, of definable objects in the language $\mathcal{L}_{\mathrm{DP}, k}$. Objects of $\operatorname{Def}_{k}$ are called definable subassignments (or definable sets). They are defined by formulas in $\mathcal{L}_{\mathrm{DP}, k}$, as follows. Let $\varphi$ be a formula in the language $\mathcal{L}_{\mathrm{DP}, k}$ having, respectively, $m, n$ and $r$ free variables in the various sorts. To such a formula $\varphi$, we assign, for every field $K$ containing $k$, the subset $h_{\varphi}(K)$ of $K((t))^{m} \times K^{n} \times \mathbb{Z}^{r}$ consisting of all points satisfying $\varphi$. An object $S$ of $\operatorname{Def}_{k}$ consists of the datum of such subsets $h_{\varphi}(K)$ for all $K$ for some $\varphi$. In particular, we may set $S(K)=h_{\varphi}(K)$. There is also a global variant GDef $_{k}$ of Def ${ }_{k}$, whose objects are definable subassignments of algebraic varieties defined over $k$, which is defined similarly via affine charts. For any $S$ in $\operatorname{Def}_{k}$ or in $\operatorname{GDef}_{k}$, a ring $\mathcal{C}(S)$ of constructible motivic functions is constructed in [CL08], and the main achievement of that paper is the construction of a theory of integration for such functions.

2.2.2 The framework we shall use. In fact, we will not deal directly with $\operatorname{Def}_{k}\left(\mathcal{L}_{\mathrm{DP}}\right)$ in the present paper, but instead we will work with a variant considered in [CL08, § 16.2]. Namely, let $T_{\text {acf }}$ be the theory of algebraically closed fields containing $k$. We shall work in the category denoted by $\operatorname{Def}_{k}\left(\mathcal{L}_{\mathrm{DP}}, T_{\text {acf }}\right)$ in loc. cit. Concretely, an object in $\operatorname{Def}_{k}\left(\mathcal{L}_{\mathrm{DP}}, T_{\text {acf }}\right)$ is obtained by evaluating an object $\operatorname{Def}_{k}$ only at algebraically closed fields containing $k$ : an object $S$ of $\operatorname{Def}_{k}\left(\mathcal{L}_{\mathrm{DP}}, T_{\text {acf }}\right)$ consists of the datum of the subsets $S(K)=h_{\varphi}(K)$ for all algebraically closed $K$ containing $k$, for some formula $\varphi$ as above. By quantifier elimination for $T_{\text {acf }}$ in $\mathcal{L}_{\mathrm{DP}, k}$, for every such $S$ there exists a formula $\varphi$ which is quantifier free. This feature shows an important difference between $\operatorname{Def}_{k}\left(\mathcal{L}_{\mathrm{DP}}, T_{\mathrm{acf}}\right)$ and $\operatorname{Def}_{k}$, since for objects in the latter category, it is in general not possible to have quantifier-free formulas in the residue field variables. The present paper being geometric in nature, we can work in $\operatorname{Def}_{k}\left(\mathcal{L}_{\mathrm{DP}}, T_{\text {acf }}\right)$, but for more arithmetical questions, one would have to stick to $\operatorname{Def}_{k}$. To shorten notation, we shall write $\operatorname{Def}_{\text {acf, }, k}$ instead of $\operatorname{Def}_{k}\left(\mathcal{L}_{\mathrm{DP}}, T_{\mathrm{acf}}\right)$ and $\mathrm{GDef}_{\text {acf }, k}$ instead of $\operatorname{GDef}_{k}\left(\mathcal{L}_{\mathrm{DP}}, T_{\mathrm{acf}}\right)$. Similarly, if $S$ is an object of $\operatorname{Def}_{\text {acf }, k}$, we shall write $\mathcal{C}_{\text {acf }}(S)$ instead of $\mathcal{C}\left(S,\left(\mathcal{L}_{\mathrm{DP}}, T_{\text {acf }}\right)\right)$, etc.

To any algebraic variety $X$ over $k((t))$ corresponds the object $\underline{X}$ in GDef $_{\text {acf }, k}$ defined by 


\section{F. LOESER AND D. WYSS}

$\underline{X}(K)=X(K((t)))$, and $X \mapsto \underline{X}$ is a functor. Similarly, there is a functor $X \mapsto \underline{X}$ from algebraic

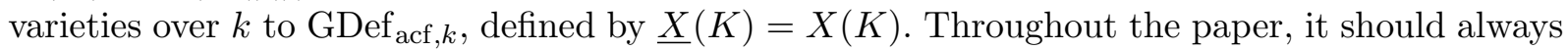
be clear whether a given algebraic variety is defined over $k((t))$ or $k$, to avoid any risk of confusion. Finally, if $X$ is defined over $k \llbracket t \rrbracket$, we will write $\underline{X}_{\circ}$ for the assignment $\underline{X}_{\circ}(K)=X(K \llbracket t \rrbracket)$.

2.2.3 Evaluation of functions. Let $S$ be in GDef $_{\text {acf, }, k}$, and let $K$ be an algebraically closed field containing $k$. For $x$ in $S(K)$, we denote by $k(x)$ the field generated by $k$, by the coefficients of the valued field components of $x$ and by the residue field components of $x$ (in the global case, one reduces to the affine case). Note that for any algebraically closed field $K^{\prime}$ containing $k(x)$, $x \in S\left(K^{\prime}\right)$ by quantifier elimination and that the field $k(x)$ is independent of the choice of $K$. We fix a Grothendieck universe $\mathcal{U}$ containing $k$, and we define the set of points $|S|$ as the colimit of the sets $S(K)$ where $K$ belongs to the category of fields extensions of $k$ in $\mathcal{U}$. (Note that this definition is slightly different from the one given in [CL08].)

Let $*_{k}$ be the terminal object in GDef $_{\text {acf }, k}$. Its set of points $\left|*_{k}\right|$ consists of a unique point $*_{k}$ with $k\left(*_{k}\right)=k$. By construction,

$$
\mathcal{C}_{\text {acf }}\left(*_{k}\right)=\mathcal{M}_{k} \otimes_{\mathbb{Z}\left[\mathbb{L}, \mathbb{L}^{-1}\right]} \mathbb{A}=K_{0}\left(\operatorname{Var}_{k}\right) \otimes_{\mathbb{Z}[\mathbb{L}]} \mathbb{A}
$$

with $\mathbb{A}=\mathbb{Z}\left[\mathbb{L}, \mathbb{L}^{-1},\left(1 /\left(1-\mathbb{L}^{-i}\right)\right)_{i>0}\right]$.

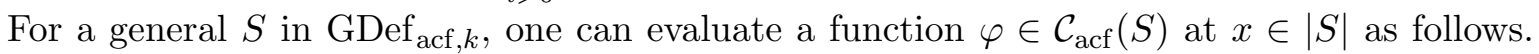
We have an inclusion morphism $i_{x}: x \hookrightarrow S \otimes k(x)$ in GDef acf,$k(x)_{\text {, where }} S \otimes k(x)$ is obtained from $S$ by extension of scalars and we identify $x$ with the terminal object in GDef acf,$k(x)_{\text {. The }}$ function $\varphi$ gives rise by base change to a function in $\mathcal{C}_{\text {acf }}(S \otimes k(x))$, and one defines $\varphi(x)$ as its pullback under the inclusion morphism $i_{x}$. It is an element of $\mathcal{M}_{k(x)} \otimes_{\mathbb{Z}\left[\mathbb{L}, \mathbb{L}^{-1}\right]} \mathbb{A}$. It is proved in [CH20] that $\varphi=0$ if and only $\varphi(x)=0$ for every point $x$ in $|S|$.

2.2.4 A Fubini theorem. Let $\varphi$ be in $\mathcal{C}_{\text {acf }}(S)$ with $S$ in Def acf, $k$ of $K$-dimension $d$ in the terminology of [CL08]. We denote by $\left|\omega_{0}\right|_{S}$ the canonical volume form in the sense of [CL08, $\S 15.1]$. As in [CL08, $\S 6.2]$, denote by $C_{\text {acf }}^{d}(S)$ the quotient of $\mathcal{C}_{\text {acf }}(S)$ by the ideal of functions with support contained in a definable subset of $K$-dimension at most $d-1$. When the class $[\varphi]$ of $\varphi$ in $C_{\text {acf }}^{d}(S)$ is integrable, the integral $\int_{S}[\varphi]\left|\omega_{0}\right|_{S}$ is defined in [CL08, $\left.\S 15.1\right]$. It belongs to $\mathcal{C}_{\text {acf }}\left(*_{k}\right)$. We shall say that $\varphi$ is integrable if its class $[\varphi]$ in $C_{\text {acf }}^{d}(S)$ is, and we shall set

$$
\int_{S} \varphi\left|\omega_{0}\right|_{S}:=\int_{S}[\varphi]\left|\omega_{0}\right|_{S}
$$

Note that if $\varphi$ is zero outside a definable subset of $K$-dimension at most $d-1$, then $\int_{S} \varphi\left|\omega_{0}\right|_{S}=0$.

Similarly, let $X$ be a smooth algebraic variety over $k((t))$ of pure dimension $d$. Let $\omega_{X}$ be a degree $d$ algebraic differential form on $X$ and $\varphi \in \mathcal{C}_{\text {acf }}(\underline{X})$. We shall say that $\varphi\left|\omega_{X}\right|$ is integrable if $[\varphi]\left|\omega_{X}\right|$ is integrable, and we set $\int_{\underline{X}} \varphi\left|\omega_{X}\right|:=\int_{\underline{X}}[\varphi]\left|\omega_{X}\right|$.

We shall use the following statement, which follows directly from Theorem 10.1.1, Proposition 15.4.1 and Section 16 of [CL08].

Proposition 2.2.5. (i) Let $X$ and $Y$ be smooth algebraic varieties over $k((t))$ of pure dimension. Let $f: X \rightarrow Y$ be a smooth morphism. We denote by $f: \underline{X} \rightarrow \underline{Y}$ the morphism induced by $f$. Let $\omega_{X}$ and $\omega_{Y}$ be everywhere non-zero top-degree algebraic differential forms on $X$ and $Y$, respectively. Let $\varphi \in \mathcal{C}_{\text {acf }}(\underline{X})$ be such that $\varphi\left|\omega_{X}\right|$ is integrable. For any point $y$ in $\underline{Y}$, denote by $\underline{X}_{y}$ the fibre of $\underline{f}$ at $y$. Then there exist a definable subset $Z$ of $\underline{Y}$ of $K$-dimension at most $\operatorname{dim} Y-1$ and a constructible motivic function $\psi \in \mathcal{C}_{\text {acf }}(\underline{Y})$ with $\psi\left|\omega_{Y}\right|$ integrable such that for 


\section{Motivic integration On the Hitchin FiBRAtion}

any point $y$ in $\underline{Y} \backslash Z$, the form $\varphi_{\mid \underline{X}_{y}}\left|\omega_{X} / f^{*}\left(\omega_{Y}\right)\right|_{\mid X_{y}}$ is integrable on $\underline{X}_{y}$ and

$$
\psi(y)=\int_{\underline{X}_{y}} \varphi_{\mid \underline{X}_{y}}\left|\omega_{X} / f^{*}\left(\omega_{Y}\right)\right|_{\mid \underline{X}_{y}}
$$

and that

$$
\int_{\underline{X}} \varphi\left|\omega_{X}\right|=\int_{\underline{Y}} \psi\left|\omega_{Y}\right|
$$

(ii) Let $X$ be a smooth algebraic variety over $k((t))$ of pure dimension. Let $Y$ be an algebraic variety over $k$. Let $\underline{f}: \underline{X} \rightarrow \underline{Y}$ be a morphism in GDef $_{\text {acf }, k}$. Let $\omega_{X}$ be a top-degree form on $X$ which is everywhere non-zero. Let $\varphi \in \mathcal{C}_{\text {acf }}(\underline{X})$ be such that $\varphi\left|\omega_{X}\right|$ is integrable. Then for any point $y$ in $\underline{Y}$, the form $\varphi_{\mid \underline{X}_{y}}\left|\omega_{X}\right|_{\mid X_{y}}$ is integrable on $\underline{X}_{y}$, and there exists a constructible motivic function $\psi \in \mathcal{C}_{\text {acf }}(\underline{Y})$ such that

$$
\psi(y)=\int_{\underline{X}_{y}} \varphi_{\mid \underline{X}_{y}}\left|\omega_{X}\right|_{\mid \underline{X}_{y}}
$$

for every point $y$ of $\underline{Y}$ and

$$
\int_{\underline{X}} \varphi\left|\omega_{X}\right|=\int_{\underline{Y}} \psi
$$

\subsection{Motivic functions with values in motives}

For $S$ in GDef $_{\text {acf }, k}$, we have a natural morphism

$$
\vartheta_{S}: \mathcal{C}_{\text {acf }}(S) \longrightarrow \prod_{x \in|S|} K_{0}\left(\mathrm{DA}_{\mathrm{ct}}^{\mathrm{ét}}(k(x), \Lambda)\right) \otimes_{\mathbb{Z}\left[\mathbb{L}, \mathbb{L}^{-1}\right]} \mathbb{A}
$$

sending a motivic function $\varphi$ to $\left(\chi_{k(x), c}(\varphi(x))\right)$. We set $\mathcal{C}_{\text {mot }}(S):=\vartheta_{S}\left(\mathcal{C}_{\text {acf }}(S)\right)$ and still denote by $\vartheta_{S}$ the induced morphism $\vartheta_{S}: \mathcal{C}_{\text {acf }}(S) \rightarrow \mathcal{C}_{\text {mot }}(S)$.

Now, let $X$ be a quasi-projective variety over $k$. In this case, $\mathcal{C}_{\text {acf }}(\underline{X})$ can be canonically identified with $\mathcal{M}_{X} \otimes_{\mathbb{Z}\left[\mathbb{L}, \mathbb{L}^{-1}\right]} \mathbb{A}$. Consider the morphism

$$
\chi_{X, c} \otimes \mathbb{A}: \mathcal{M}_{X} \otimes_{\mathbb{Z}\left[\mathbb{L}, \mathbb{L}^{-1}\right]} \mathbb{A} \longrightarrow K_{0}\left(\mathrm{DA}_{\mathrm{ct}}^{\text {ét }}(X, \Lambda)\right) \otimes_{\mathbb{Z}\left[\mathbb{L}, \mathbb{L}^{-1}\right]} \mathbb{A} .
$$

It follows from Lemma 2.1.1 that $\mathcal{C}_{\text {mot }}(\underline{X})$ can be canonically identified with the image of $\chi_{X, c} \otimes \mathbb{A}$ and that under that identification, the morphisms $\vartheta_{X}: \mathcal{C}_{\text {acf }}(\underline{X}) \rightarrow \mathcal{C}_{\operatorname{mot}}(\underline{X})$ and $\chi_{X, c} \otimes \mathbb{A}$ : $\mathcal{C}_{\text {acf }}(\underline{X}) \rightarrow \mathcal{C}_{\text {mot }}(\underline{X})$ are equal.

Consider a morphism $q: X \rightarrow Y$ between quasi-projective varieties over $k$. Tensoring the morphisms $q$ ! and $q^{*}$ with $\mathbb{A}$, one gets morphisms $q_{!}: \mathcal{C}_{\text {acf }}(\underline{X}) \rightarrow \mathcal{C}_{\text {acf }}(\underline{Y})$ and $q^{*}: \mathcal{C}_{\text {acf }}(\underline{Y}) \rightarrow$ $\mathcal{C}_{\text {acf }}(\underline{X})$. One deduces from the commutative diagrams (2.1.1) and (2.1.2) that the morphisms $q_{!}:$ $\mathcal{M}_{X} \otimes_{\mathbb{Z}\left[\mathbb{L}, \mathbb{L}^{-1}\right]} \mathbb{A} \rightarrow \mathcal{M}_{Y} \otimes_{\mathbb{Z}\left[\mathbb{L}, \mathbb{L}^{-1}\right]} \mathbb{A}$ and $q^{*}: \mathcal{M}_{Y} \otimes_{\mathbb{Z}\left[\mathbb{L}, \mathbb{L}^{-1}\right]} \mathbb{A} \rightarrow \mathcal{M}_{X} \otimes_{\mathbb{Z}\left[\mathbb{L}, \mathbb{L}^{-1}\right]} \mathbb{A}$ obtained by tensoring with $\mathbb{A}$ induce morphisms $q_{!}: \mathcal{C}_{\text {mot }}(\underline{X}) \rightarrow \mathcal{C}_{\text {mot }}(\underline{Y})$ and $q^{*}: \mathcal{C}_{\text {mot }}(\underline{Y}) \rightarrow \mathcal{C}_{\text {mot }}(\underline{X})$ and that the diagrams

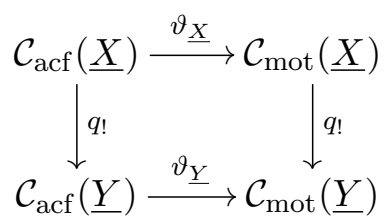




\section{F. LOESER AND D. Wyss}

and

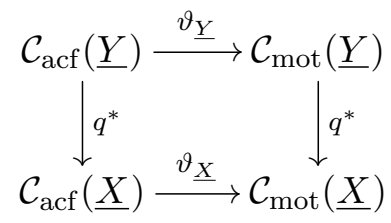

commute.

A crucial property of $\vartheta_{S}$ is that it commutes with integration.

Proposition-Definition 2.3.1. Let $S$ be in $\operatorname{Def}_{\text {acf }, k}$. Let $\varphi$ and $\varphi^{\prime}$ be in $\mathcal{C}_{\text {acf }}(S)$. Assume that $\varphi$ and $\varphi^{\prime}$ are integrable and that we have $\vartheta_{S}(\varphi)=\vartheta_{S}\left(\varphi^{\prime}\right)$. Then

$$
\vartheta_{*_{k}}\left(\int_{S} \varphi\left|\omega_{0}\right|_{S}\right)=\vartheta_{*_{k}}\left(\int_{S} \varphi^{\prime}\left|\omega_{0}\right|_{S}\right)
$$

in $\mathcal{C}_{\text {mot }}\left(*_{k}\right)$ (and thus also in $K_{0}\left(\mathrm{M}_{\text {rat }}(k, \Lambda)\right) \otimes_{\mathbb{Z}\left[\mathbb{L}, \mathbb{L}^{-1}\right]} \mathbb{A}$ ). In this case, we say that $\vartheta_{S}(\varphi)$ is integrable, and we set

$$
\int_{S} \vartheta_{S}(\varphi)\left|\omega_{0}\right|_{S}:=\vartheta_{*_{k}}\left(\int_{S} \varphi\left|\omega_{0}\right|_{S}\right)
$$

Proof. The construction of the motivic integral in [CL08] is performed in several steps. It uses cell decomposition to reduce to one of the following three cases: (i) integration over cells in one variable in the valued field sort in the sense of [CL08, §7.1], (ii) integration over residue field variables and (iii) integration over value group variables. Integration over cells in one variable in the valued field sort uses pullbacks over residue field variables and value group variables. Once one notices that pullback over residue field variables commutes with $\vartheta$ by the commutativity of diagram (2.3.2) and that pullback over value group variables obviously commutes with $\vartheta$, it is clear that integration in case (i) commutes with $\vartheta$. The commutation of integration in case (ii) with $\vartheta$ follows directly from the commutativity of diagram (2.3.1), while the commutation of integration in case (iii) with $\vartheta$ is clear.

Let $X$ be a smooth algebraic variety over $k((t))$ of pure dimension, and let $\omega_{X}$ be a top-degree form on $X$. Let $\varphi \in \mathcal{C}_{\text {acf }}(\underline{X})$ be such that $\varphi\left|\omega_{X}\right|$ is integrable. Using affine charts, one deduces from Proposition 2.3.1 that $\vartheta_{*_{k}}\left(\int_{\underline{X}} \varphi\left|\omega_{X}\right|_{S}\right)$ depends only on $\vartheta_{\underline{X}}(\varphi)$. In this case, we say that $\vartheta_{X}(\varphi)\left|\omega_{X}\right|$ is integrable, and we set

$$
\int_{\underline{X}}^{\mathrm{mot}} \varphi\left|\omega_{X}\right|:=\vartheta_{*_{k}}\left(\int_{\underline{X}} \varphi\left|\omega_{X}\right|_{S}\right) .
$$

Similarly, if $Y$ is an algebraic variety over $k$ and $\varphi \in \mathcal{C}_{\text {acf }}(\underline{Y})$, one sets

$$
\int_{\underline{Y}}^{\text {mot }} \varphi:=\vartheta_{*_{k}}\left(\int_{\underline{Y}} \varphi\right) \text {. }
$$

In particular, we have the following consequence of Proposition 2.2.5.

Proposition 2.3.2. (i) Let $X$ and $Y$ be smooth algebraic varieties over $k((t))$ of pure dimension. Let $f: X \rightarrow Y$ be a smooth morphism. Let $\omega_{X}$ and $\omega_{Y}$ be everywhere non-zero top-degree forms on $X$ and $Y$, respectively. Let $\varphi \in \mathcal{C}_{\text {acf }}(\underline{X})$ be such that $\varphi\left|\omega_{X}\right|$ is integrable. Then there exist a definable subset $Z$ of $\underline{Y}$ of $K$-dimension at most $\operatorname{dim} Y-1$ and a $\psi \in \mathcal{C}_{\text {acf }}(\underline{Y})$ with $\psi\left|\omega_{Y}\right|$ 


\section{Motivic INTEGRATion ON THE Hitchin FibRATion}

integrable such that for any point $y$ in $\underline{Y} \backslash Z$, the form $\vartheta_{\underline{X}_{y}}\left(\varphi_{\mid \underline{X}_{y}}\right)\left|\omega_{X} / f^{*}\left(\omega_{Y}\right)\right|_{\mid \underline{X}_{y}}$ is integrable,

$$
\vartheta_{\underline{Y}}(\psi)(y)=\int_{\underline{X}_{y}}^{\text {mot }} \varphi_{\underline{X}_{y}}\left|\omega_{X} / f^{*}\left(\omega_{Y}\right)\right|_{\underline{X}_{y}},
$$

and

$$
\int_{\underline{X}}^{\text {mot }} \varphi\left|\omega_{X}\right|=\int_{\underline{Y}}^{\text {mot }} \psi\left|\omega_{Y}\right| .
$$

(ii) Let $X$ be a smooth algebraic variety over $k((t))$ of pure dimension. Let $Y$ be an algebraic

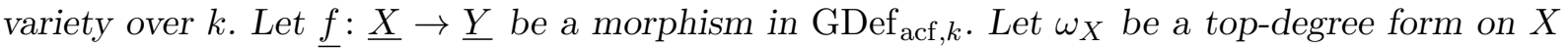
which is everywhere non-zero. Let $\varphi \in \mathcal{C}_{\text {acf }}(\underline{X})$ be such that $\varphi\left|\omega_{X}\right|$ is integrable. Then, for any point $y$ in $\underline{Y}$, the form $\vartheta_{\underline{X}_{y}}\left(\varphi_{\mid \underline{X}_{y}}\right)\left|\omega_{X}\right|_{\underline{X}_{y}}$ is integrable and there exists a $\psi \in \mathcal{C}_{\text {acf }}(\underline{Y})$ such that

$$
\vartheta_{\underline{Y}}(\psi)(y)=\int_{\underline{X}_{y}}^{\operatorname{mot}} \varphi_{\mid \underline{X}_{y}}\left|\omega_{X}\right|_{\mid \underline{X}_{y}}
$$

for every point $y$ of $\underline{Y}$ and

$$
\int_{\underline{X}}^{\text {mot }} \varphi\left|\omega_{X}\right|=\int_{\underline{Y}}^{\text {mot }} \psi
$$

\subsection{The equivariant case}

We fix a finite commutative group $\Gamma$. Let $S$ be an object of $\operatorname{GDef}_{\text {acf, }, k}$. Recall that the $\operatorname{ring} \mathcal{C}_{\text {acf }}(S)$ of constructible motivic functions is defined in Section 5.3 of [CL08] as a tensor product

$$
\mathcal{C}_{\text {acf }}(S):=K_{0}\left(\operatorname{RDef}_{\text {acf }, S}\right) \otimes_{\mathcal{P}^{0}(S)} \mathcal{P}(S) .
$$

Here the ring $\mathcal{P}(S)$ is the ring of Presburger functions defined in Section 4.6 of [CL08], $\mathcal{P}^{0}(S)$ is a subring of $\mathcal{P}(S)$ defined in Section 5.2 of [CL08], and $K_{0}\left(\mathrm{RDef}_{\mathrm{acf}, S}\right)$ is the Grothendieck ring of

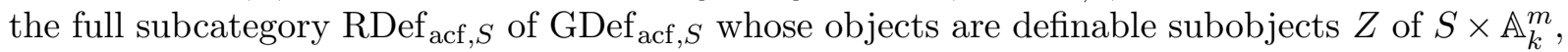
for some $m$, where, by abuse of notation, we denote by $\mathbb{A}_{k}^{m}$ the object whose $K$-points are $K^{m}$, for every field extension $K$ of $k$. For such a $Z$, denote the projection to $S$ by $p_{Z}: Z \rightarrow S$.

We define $\operatorname{RDef}_{\mathrm{acf}, S}^{\Gamma}$ as the category whose objects are objects $Z$ of $\operatorname{RDef}_{\text {acf, } S}$ endowed with a definable $\Gamma$-action $\Gamma \times Z \rightarrow Z$ leaving the fibres of $p_{Z}$ stable and satisfying the following condition: for any point $x$ of $S$, there exists a finite partition of the fibre $Z_{x}$ into Zariski-locally closed subsets $W_{i}$ which are stable under the $\Gamma$-action, and the $\Gamma$-action $\Gamma \times W_{i} \rightarrow W_{i}$ is algebraic (viewing $\Gamma$ as a finite group scheme). Morphisms in $\operatorname{RDef}_{\text {acf }, S}^{\Gamma}$ are $\Gamma$-equivariant morphisms in $\operatorname{RDef}_{\text {acf }, S}$. We have a morphism $\mathcal{P}^{0}(S) \rightarrow K_{0}\left(\operatorname{RDef}_{\text {acf, } S}^{\Gamma}\right)$ obtained by composing the morphism $\mathcal{P}^{0}(S) \rightarrow K_{0}\left(\operatorname{RDef}_{\text {acf }, S}\right)$ with the morphism $K_{0}\left(\operatorname{RDef}_{\text {acf }, S}\right) \rightarrow K_{0}\left(\operatorname{RDef}_{\text {acf }, S}^{\Gamma}\right)$, which is induced by the functor sending an object in $\operatorname{RDef}_{\text {acf }, S}$ to the same object endowed with the trivial $\Gamma$ action. We define the ring $\mathcal{C}_{\text {acf }}^{\Gamma}(S)$ as the tensor product $\mathcal{C}_{\text {acf }}^{\Gamma}(S):=K_{0}\left(\operatorname{RDef}_{\text {acf }, S}^{\Gamma}\right) \otimes_{\mathcal{P}^{0}(S)} \mathcal{P}(S)$.

The integration theory developed in [CL08] for $\mathcal{C}_{\text {acf }}(S)$ can be reproduced verbatim for $\mathcal{C}_{\text {acf }}^{\Gamma}(S)$. In particular, we have the following equivariant version of Proposition 2.3.2.

Proposition 2.4.1. The statement of Proposition 2.3.2 holds with $\mathcal{C}_{\text {acf }}$ replaced by $\mathcal{C}_{\text {acf }}^{\Gamma}$.

We have a natural functor $q: \operatorname{RDef}_{\text {acf }, S}^{\Gamma} \rightarrow \operatorname{RDef}_{\text {acf }, S}$ which sends an object $X$ in $\operatorname{RDef}_{\text {acf }, S}^{\Gamma}$ to the quotient $X / \Gamma$. It induces a map $q: K_{0}\left(\operatorname{RDef}_{\text {acf }, S}^{\Gamma}\right) \rightarrow K_{0}\left(\operatorname{RDef}_{\text {acf }, S}\right)$ which can be extended by linearity to a map $q: \mathcal{C}_{\text {acf }}^{\Gamma}(S) \rightarrow \mathcal{C}_{\text {acf }}(S)$. 


\section{F. LOESER AND D. WYSS}

Proposition 2.4.2. Let $X$ be a smooth algebraic variety of pure dimension over $k((t))$, and let $\omega$ be a top-degree form. Let $S \subset \underline{X}$ be in $\operatorname{GDef}_{\text {acf }, k}$. Let $\psi \in \mathcal{C}_{\text {acf }}^{\Gamma}(S)$ be integrable. We have

$$
q\left(\int_{S} \psi|\omega|\right)=\int_{S} q(\psi)|\omega|
$$

Proof. The commutation of $q$ with integration is clear at each step in the construction of the motivic integral.

Now let $S$ be a quasi-projective variety over $k$. We denote by $\operatorname{Var}_{S}^{\Gamma}$ the category of morphisms $p: X \rightarrow S$ with $X$ quasi-projective and endowed with an algebraic $\Gamma$-action such that for every point $x$ of $X$, the $\Gamma$-orbit of $x$ is contained in an affine subset of $p^{-1}(p(x))$. The morphisms are $S$-morphisms equivariant with respect to the $\Gamma$-action. We denote by $K_{0}\left(\operatorname{Var}_{S}^{\Gamma}\right)$ the corresponding Grothendieck ring of varieties over $S$ and by $\mathcal{M}_{S}^{\Gamma}$ its localization by the class $\mathbb{L}$ of the affine line over $S$ endowed with the trivial $\Gamma$-action.

If $q: S \rightarrow T$ is a morphism of quasi-projective $k$-schemes, composition with $q$ and pullback induce, respectively, morphisms

$$
\begin{aligned}
q ! & \mathcal{M}_{S}^{\Gamma} \longrightarrow \mathcal{M}_{T}^{\Gamma} \\
q^{*}: \mathcal{M}_{T}^{\Gamma} & \longrightarrow \mathcal{M}_{S}^{\Gamma} .
\end{aligned}
$$

We assume from now on that $\Lambda$ is the field $\mathbb{Q}\left(\mu_{r}\right)$ with $r$ the order of the group $\Gamma$, and we denote the group of characters $\Gamma \rightarrow \Lambda^{\times}$by $\widehat{\Gamma}$.

Let $S$ be a quasi-projective $k$-scheme endowed with the trivial $\Gamma$-action. Consider $M \in$ $\mathrm{DA}_{\mathrm{ct}}^{\text {ét }}(S, \Lambda)$ endowed with a $\Gamma$-action, that is, a group morphism $a: \Gamma \rightarrow \operatorname{Aut}_{\mathrm{DA}_{\mathrm{ct}}^{\text {et }}(S, \Lambda)}(M)$. Fix $\delta \in \widehat{\Gamma}$, and set $p_{\delta}:=|\Gamma|^{-1} \sum_{\gamma \in \Gamma} \delta^{-1}(\gamma) a(\gamma)$. The morphism $p_{\delta}$ is a projector. Since $\Lambda$ is a $\mathbb{Q}$ vector space, the category $\operatorname{DA}_{\mathrm{ct}}^{\text {et }}(S, \Lambda)$ is pseudo-abelian; cf. [Ayo14b, Proposition 9.2]. Thus we can consider the image of $p_{\delta}$ as an object in $\operatorname{DA}_{\mathrm{ct}}^{\text {et }}(S, \Lambda)$, which we denote by $M^{\delta}$ and call the $\delta$-isotypical component of $M$.

Let $p: X \rightarrow S$ be a quasi-projective $S$-scheme endowed with an algebraic $\Gamma$-action such that for every point $x$ of $X$, the $\Gamma$-orbit of $x$ is contained in an affine subset of $p^{-1}(p(x))$. By functoriality, the object $p_{!}\left(\mathbb{1}_{X}\right)$ is endowed with a $\Gamma$-action. Thus we can consider its $\delta$-isotypical component $p_{!}\left(\mathbb{1}_{X}\right)^{\delta}$.

By the same argument as that in [IS13, Lemma 2.1], there is a unique group morphism

$$
\chi_{S, c}^{\delta}: \mathcal{M}_{S}^{\Gamma} \longrightarrow K_{0}\left(\mathrm{DA}_{\mathrm{ct}}^{\mathrm{ét}}(S, \Lambda)\right)
$$

such that for $p: X \rightarrow S$ as above, $\chi_{S, c}^{\delta}([X]):=\left[p_{!}\left(\mathbb{1}_{X}\right)^{\delta}\right]$. Furthermore, the diagrams

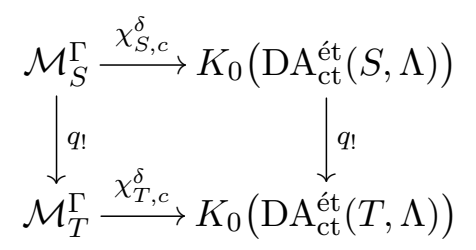


and

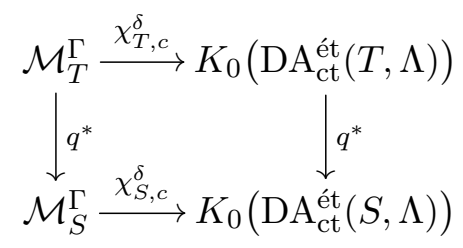

are commutative.

For $S$ in GDef $_{\text {acf, }, k}$, we have a natural morphism

$$
\vartheta_{S}^{\delta}: \mathcal{C}_{\mathrm{acf}}^{\Gamma}(S) \longrightarrow \prod_{x \in|S|} K_{0}\left(\mathrm{DA}_{\mathrm{ct}}^{\mathrm{et}}(k(x), \Lambda)\right) \otimes_{\mathbb{Z}\left[\mathbb{L}, \mathbb{L}^{-1}\right]} \mathbb{A}
$$

sending $\varphi$ to $\left(\chi_{k(x), c}^{\delta}(\varphi(x))\right)$. We denote by $\mathcal{C}_{\text {mot }}^{\Gamma}(S)$ the subring of $K_{0}\left(\mathrm{DA}_{\mathrm{ct}}^{\mathrm{et}}(k(x), \Lambda)\right) \otimes_{\mathbb{Z}\left[\mathbb{L}, \mathbb{L}^{-1}\right]} \mathbb{A}$ generated by the images $\vartheta_{S}^{\delta}\left(\mathcal{C}_{\text {acf }}(S)\right)$, for $\delta$ running over $\widehat{\Gamma}$. Note that $\vartheta_{S}(\varphi)=\sum_{\delta \in \widehat{\Gamma}} \vartheta_{S}^{\delta}(\varphi)$ in $\mathcal{C}_{\text {mot }}^{\Gamma}(S)$.

If $X$ is a quasi-projective variety over $k$, then $\mathcal{C}_{\text {acf }}^{\Gamma}(\underline{X})$ can be canonically identified with $\mathcal{M}_{X}^{\Gamma} \otimes_{\mathbb{Z}\left[\mathbb{L}, \mathbb{L}^{-1}\right]} \mathbb{A}$. For any character $\delta$, we have a morphism

$$
\chi_{X, c}^{\delta} \otimes \mathbb{A}: \mathcal{M}_{X}^{\Gamma} \otimes_{\mathbb{Z}\left[\mathbb{L}, \mathbb{L}^{-1}\right]} \mathbb{A} \longrightarrow K_{0}\left(\mathrm{DA}_{\mathrm{ct}}^{\mathrm{ét}}(X, \Lambda)\right) \otimes_{\mathbb{Z}\left[\mathbb{L}, \mathbb{L}^{-1}\right]} \mathbb{A} .
$$

Again, it follows from Lemma 2.1.1 that $\mathcal{C}_{\text {mot }}^{\Gamma}(\underline{X})$ can be canonically identified with the subring generated by the images of $\chi_{X, c}^{\delta} \otimes \mathbb{A}$ and that under that identification, $\vartheta_{\underline{X}}^{\delta}$ and $\chi_{X, c}^{\delta} \otimes \mathbb{A}$ define the same morphisms $\mathcal{C}_{\text {acf }}^{\Gamma}(\underline{X}) \rightarrow \mathcal{C}_{\text {mot }}^{\Gamma}(\underline{X})$.

As in the non-equivariant case, given a morphism $q: X \rightarrow Y$ between quasi-projective varieties over $k$, one constructs morphisms $q_{!}: \mathcal{C}_{\text {mot }}^{\Gamma}(\underline{X}) \rightarrow \mathcal{C}_{\text {mot }}^{\Gamma}(\underline{Y})$ and $q^{*}: \mathcal{C}_{\text {mot }}^{\Gamma}(\underline{Y}) \rightarrow \mathcal{C}_{\text {mot }}^{\Gamma}(\underline{X})$ such that the diagrams

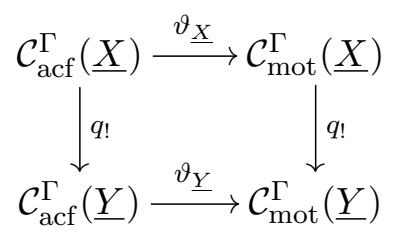

and

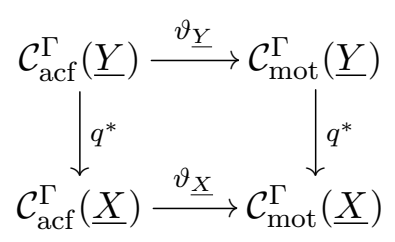

commute.

Let $S$ be in Def acf,k, and consider an integrable function $\varphi$ in $\mathcal{C}_{\text {acf }}^{\Gamma}(S)$. One deduces, as in Proposition 2.3.1, that $\vartheta_{*_{k}}^{\delta}\left(\int_{S} \varphi\left|\omega_{0}\right|_{S}\right)$ depends only on $\vartheta_{S}^{\delta}(\varphi)$, and we denote it by $\int_{S}^{\text {mot, } \delta} \varphi\left|\omega_{0}\right|_{S}$. This extends to the global case as above: if $X$ is a smooth algebraic variety over $k((t))$ of pure dimension, $\omega_{X}$ a top-degree form on $X$ and $\varphi$ a function in $\mathcal{C}_{\text {acf }}^{\Gamma}(\underline{X})$ such that $\varphi\left|\omega_{X}\right|$ is integrable, 


\section{F. LOESER AND D. WYSS}

then $\vartheta_{*_{k}}^{\delta}\left(\int_{\underline{X}} \varphi\left|\omega_{X}\right| S\right)$ depends only on $\vartheta_{\underline{X}}^{\delta}(\varphi)$ and we denote it by $\int_{\underline{X}}^{\text {mot, }, \delta} \varphi\left|\omega_{X}\right|$. We will also set

$$
\int_{\underline{X}}^{\mathrm{mot}} \varphi\left|\omega_{X}\right|=\sum_{\delta \in \widehat{\Gamma}} \int_{\underline{X}}^{\mathrm{mot}, \delta} \varphi\left|\omega_{X}\right| .
$$

If $Y$ is an algebraic variety over $k$ and $\varphi$ is a function in $\mathcal{C}_{\text {acf }}^{\Gamma}(\underline{Y})$, one defines similarly $\int_{\underline{Y}}^{\text {mot, } \delta} \varphi$ and $\int_{\underline{Y}}^{\operatorname{mot}} \varphi$.

We may now state the following final avatar of Proposition 2.2.5 which we will use in this paper. It follows directly from Proposition 2.4.1 and the above discussion.

Proposition 2.4.3. (i) Let $X$ and $Y$ be smooth algebraic varieties over $k((t))$ of pure dimension. Let $f: X \rightarrow Y$ be a smooth morphism. Let $\omega_{X}$ and $\omega_{Y}$ be everywhere non-zero top-degree forms on $X$ and $Y$, respectively. Let $\varphi \in \mathcal{C}_{\text {acf }}(\underline{X})$ be such that $\varphi\left|\omega_{X}\right|$ is integrable. Let $\delta$ be in $\widehat{\Gamma}$. There exist a definable subset $Z$ of $\underline{Y}$ of $K$-dimension at most $\operatorname{dim} Y-1$ and a $\psi \in \mathcal{C}_{\text {acf }}^{\Gamma}(\underline{Y})$ with $\psi\left|\omega_{Y}\right|$ integrable such that

$$
\vartheta_{\underline{Y}}^{\delta}(\psi)(y)=\int_{\underline{X}_{y}}^{\text {mot, } \delta} \varphi_{\mid \underline{X}_{y}}\left|\omega_{X} / f^{*}\left(\omega_{Y}\right)\right|_{\underline{X}_{y}}
$$

for every point $y$ of $\underline{Y} \backslash Z$ and

$$
\int_{\underline{X}}^{\mathrm{mot}, \delta} \varphi\left|\omega_{X}\right|=\int_{\underline{Y}}^{\mathrm{mot}, \delta} \psi\left|\omega_{Y}\right| .
$$

(ii) Let $X$ be a smooth algebraic variety over $k((t))$ of pure dimension. Let $Y$ be an algebraic

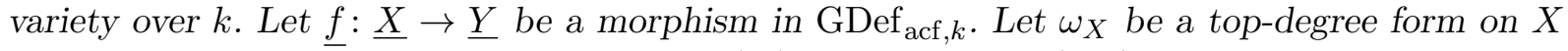
which is everywhere non-zero. Let $\varphi \in \mathcal{C}_{\text {acf }}(\underline{X})$ be such that $\varphi\left|\omega_{X}\right|$ is integrable. Then, there exists a $\psi \in \mathcal{C}_{\text {acf }}^{\Gamma}(\underline{Y})$ such that

$$
\vartheta_{\underline{Y}}^{\delta}(\psi)(y)=\int_{\underline{X}_{y}}^{\text {mot, } \delta} \varphi_{\mid \underline{X}_{y}}\left|\omega_{X}\right|_{\mid \underline{X}_{y}}
$$

for every point $y$ of $\underline{Y}$ and

$$
\int_{\underline{X}}^{\mathrm{mot}, \delta} \varphi\left|\omega_{X}\right|=\int_{\underline{Y}}^{\mathrm{mot}, \delta} \psi .
$$

\subsection{Some useful facts}

We collect here some well-known facts that will be used later in the paper.

Let $X$ be a quasi-projective variety over a field of characteristic zero on which a finite group $\Gamma$ acts algebraically, and assume that for every point $x$ of $X$, the $\Gamma$-orbit of $x$ is contained in an affine subset of $X$. Let $h: X \rightarrow Y:=X / \Gamma$ be the quotient map. The morphism $\mathbb{1}_{Y} \rightarrow h_{*} h^{*}\left(\mathbb{1}_{Y}\right)=$ $h_{*}\left(\mathbb{1}_{X}\right)$ given by the unit of the adjunction induces an isomorphism

$$
\mathbb{1}_{Y} \cong h_{*}\left(\mathbb{1}_{X}\right)^{1},
$$

where $h_{*}\left(\mathbb{1}_{X}\right)^{1}$ is the isotypical part of $h_{*}\left(\mathbb{1}_{X}\right)$ for the trivial character. This can be checked directly from the definitions, or by proper base change, one can reduce to the case where $Y$ is a point, which is even simpler. Note that since $h$ is proper, one can identify $h_{*}\left(\mathbb{1}_{X}\right)$ with $h_{!}\left(\mathbb{1}_{X}\right)$.

If $\pi: X \rightarrow$ Spec $k$ is an algebraic variety over $k$, we shall consider its homological Chow motive $M(X):=\pi ! \pi^{!}\left(\mathbb{1}_{\text {Spec } k}\right)$. 


\section{Motivic INTEGRATion ON THE Hitchin FibRATion}

Lemma 2.5.1. Let $k$ be a field of characteristic zero. Let $A$ and $B$ be connected commutative algebraic groups over $k$, and let $p: A \rightarrow B$ be an isogeny. Then the morphism $M(A) \rightarrow M(B)$ induced by $p$ is an isomorphism.

Proof. It is enough to check that for any non-zero natural number $n$, the morphism $[n]: M(A) \rightarrow$ $M(A)$ induced by multiplication by $n$ is an isomorphism, which follows from Theorem 7.1.1(1) in [AEH15] in the semiabelian case and from Theorem 3.3(4) in [AHP16] in general.

Lemma 2.5.2. Let $k$ be a field of characteristic zero. Let $p: A \rightarrow B$ be an isogeny between connected commutative algebraic groups over $k$ with kernel $\Gamma$. Denote the structural morphisms by $\pi_{A}$ and $\pi_{B}$. The finite abelian group $\Gamma$ acts on the object $p_{!}\left(\mathbb{1}_{A}\right)$ of $\mathrm{DA}_{\mathrm{ct}}^{\mathrm{e}}(B, \Lambda)$, and for any character $\delta: \Gamma \rightarrow \Lambda^{\times}$, we denote its $\delta$-isotypical component by $p_{!}\left(\mathbb{1}_{A}\right)^{\delta}$. Then $\pi_{B !}\left(p_{!}\left(\mathbb{1}_{A}\right)^{\delta}\right)=0$ if $\delta$ is non-trivial and $\pi_{B !}\left(p_{!}\left(\mathbb{1}_{A}\right)^{\delta}\right)=\pi_{B !}\left(p_{!}\left(\mathbb{1}_{A}\right)\right)$ if $\delta$ is trivial.

Proof. As we already observed, the morphism

$$
\mathbb{1}_{B} \longrightarrow p_{*}\left(\mathbb{1}_{A}\right)
$$

induces an isomorphism between $\mathbb{1}_{B}$ and $p_{*}\left(\mathbb{1}_{A}\right)^{1}$. Dualizing this morphism, one gets a morphism

$$
p_{!} \pi_{A}^{!}\left(\mathbb{1}_{\text {Spec } k}\right) \longrightarrow \pi_{B}^{!}\left(\mathbb{1}_{\text {Spec } k}\right)
$$

whose restriction to $\left(p_{!} \pi_{A}^{!}\left(\mathbb{1}_{\mathrm{Spec} k}\right)\right)^{\delta}$ is zero when $\delta$ is non-trivial and an isomorphism when $\delta$ is trivial. Applying $\pi_{B}$ ! to both sides of (2.5.3), one gets a morphism $\pi_{A !} \pi_{A}^{!}\left(\mathbb{1}_{\text {Spec } k}\right) \rightarrow$ $\pi_{B !} \pi_{B}^{!}\left(\mathbb{1}_{\text {Spec } k}\right)$ which is an isomorphism by Lemma 2.5.1. It follows that $\pi_{B !}\left(\left(p_{!} \pi_{A}^{!}\left(\mathbb{1}_{\text {Spec } k}\right)\right)^{\delta}\right)$ is zero when $\delta$ is non-trivial and isomorphic to $\pi_{B !} \pi_{B}^{!}\left(\mathbb{1}_{\text {Spec } k}\right)$ when $\delta$ is trivial. The statement follows since $\pi_{A}^{!}\left(\mathbb{1}_{\text {Spec } k}\right) \simeq \mathbb{1}_{A}(d)[2 d]$ and $\pi_{B}^{!}\left(\mathbb{1}_{\text {Spec } k}\right) \simeq \mathbb{1}_{B}(d)[2 d]$, with $d$ the dimension of both $A$ and $B$.

Corollary 2.5.3. Let $k$ be a field of characteristic zero. Let $\pi: A \rightarrow \operatorname{Spec} k$ be a connected commutative algebraic group, and let $\xi \in A(k)$ be a torsion point. Denote by $g_{\xi}$ the endomorphism given by translation by $\xi$. Then $g_{\xi}$ acts trivially on $\pi_{!}\left(\mathbb{1}_{A}\right) \simeq M(A)(-d)[2 d]$.

Proof. This follows directly from Lemma 2.5.2 applied to the isogeny $A \rightarrow B$ with $B$ the quotient of $A$ by the subgroup generated by $\xi$.

\subsection{Functions from quasi-finite morphisms}

Consider a quasi-finite morphism $f: X \longrightarrow Y$ between $k((t))$-varieties of pure dimension. Then any $S \subset \underline{X}$ in GDef $_{\text {acf }, k}$ is isomorphic to an object in $\operatorname{RDef}_{\text {acf, } Y}$ by an isomorphism commuting with the map to $\underline{Y}$. Indeed, this follows from Corollary 7.2.3 of [CL08] (there is an unfortunate typo, $r$ should read 0 ).

Thus, we may attach to $S$ a constructible motivic function in $\mathcal{C}_{\text {acf }}(\underline{Y})$ (or in $\mathcal{C}_{\text {acf }}(f(S)$ ) which we still denote by $S$. Now, assume that $\Gamma$ acts on $X$, that $f$ is $\Gamma$-invariant and that $S$ is stable under $\Gamma$. We define similarly a function in $\mathcal{C}_{\text {acf }}^{\Gamma}(\underline{Y})$ (or in $\mathcal{C}_{\text {acf }}^{\Gamma}(\underline{f}(S))$ which we will also denote by $S$.

Remark 2.6.1. The support $f(S)$ of $S$ as a function is in general smaller than the subassignment associated with the image $f \overline{(S)}$. In fact, a point $x \in f(S)(K)$ belongs to $f(S)$ if the fibre $f^{-1}(x)$ admits a $K^{\prime}((t))$-rational point for some extension $\overline{K^{\prime} / K}$.

The following lemma allows us to compute with these functions. 


\section{F. LOESER AND D. WYSS}

Lemma 2.6.2. Let $f: X \longrightarrow Y$ be an étale morphism between smooth $k \llbracket t \rrbracket$-varieties, both of pure relative dimension $d$, and let $\omega$ be a degree $d$ differential form on $Y$ which generates the $k \llbracket t \rrbracket$-module of degree $d$ differential forms everywhere. Then we have

$$
\int_{\underline{Y}_{\circ}} X|\omega|=\mathbb{L}^{-d}\left[X_{k}\right]
$$

with $X_{k}$ the special fibre of $X$. If a finite abelian group $\Gamma$ acts on $X$ and $f$ is $\Gamma$-invariant, the same holds $\Gamma$-equivariantly.

Proof. Consider the definable morphism ev $\underline{\mathrm{ev}}_{X} \underline{X}_{\circ} \rightarrow \underline{X}_{k}$ given by reducing modulo $t$, and define $\underline{\mathrm{ev}_{Y}}$ similarly. The morphism $\left(\underline{f}, \mathrm{ev}_{X}\right): \underline{X}_{\circ} \rightarrow \underline{Y}_{\circ} \times \underline{X_{k}}$ is an isomorphism since $f$ is étale. Thus the function in $\mathcal{C}_{\text {acf }}\left(\underline{Y}_{\circ}\right)$ associated with $\underline{X}_{\circ}$ is of the form $\underline{\operatorname{ev}}_{Y}{ }^{*}(\varphi)$ with $\varphi$ the function $\mathcal{C}_{\text {acf }}\left(\underline{Y_{k}}\right)$ associated with $\underline{X_{k}}$. The lemma now follows directly from Lemma 3.1.1.

\subsection{Twisting}

Let $\Gamma$ and $\Gamma^{\prime}$ be two finite commutative groups. Let $S$ be an object of GDef $_{\text {acf, } k}$, and let $\varphi \in$ $\operatorname{RDef}_{\text {acf }, S}^{\Gamma \times \Gamma^{\prime}}$ and $\psi \in \operatorname{RDef}_{\text {acf, } S}^{\Gamma}$. We let $\Gamma$ act anti-diagonally on the product $\psi \times \varphi$ in $\operatorname{RDef}_{\text {acf }, S}$ by $\gamma(a, b)=\left(\gamma a, \gamma^{-1} b\right)$ and consider the quotient

$$
\psi \times \times^{\Gamma} \varphi:=\psi \times_{S} \varphi / \Gamma
$$

as an object of $\operatorname{RDef}_{\mathrm{acf}, S}^{\Gamma^{\prime}}$. This way, we get a morphism $\cdot \times^{\Gamma} \varphi: \operatorname{RDef}_{\mathrm{acf}, S}^{\Gamma} \rightarrow \operatorname{RDef}_{\mathrm{acf}, S}^{\Gamma^{\prime}}$ which extends by $\mathcal{P}(S)$-linearity to a morphism $\cdot \times^{\Gamma} \varphi: \mathcal{C}_{\text {acf }}^{\Gamma}(S) \rightarrow \mathcal{C}_{\text {acf }}^{\Gamma^{\prime}}(S)$. We state two lemmas for later use. The first explains the effect of unramified twisting on integrals.

Lemma 2.7.1. Let $X$ be a smooth algebraic variety of pure dimension over $k((t))$, and let $\omega$ be a top-degree form. Let $S \subset \underline{X}$ be in $\operatorname{GDef}_{\text {acf }, k}$. Let $\varphi \in \mathcal{C}_{\text {acf }}^{\Gamma \times \Gamma^{\prime}}\left(*_{k}\right)$ and $\psi \in \mathcal{C}_{\text {acf }}^{\Gamma}(S)$ be integrable. We have

$$
\int_{S} \psi \times \times^{\Gamma} p^{*}(\varphi)|\omega|=\left(\int_{S} \psi|\omega|\right) \times \times^{\Gamma} \varphi
$$

where $p: S \rightarrow *_{k}$ is the morphism to the point.

Proof. This follows directly from the projection formula in Proposition 13.2.1(2) in [CL08] and Proposition 2.4.2.

The second lemma is about the effect of twisting on isotypical components. For this, we assume $S$ to be a finite-type $k((t))$-scheme and $T$ a $\Gamma$-torsor on $S$. We can consider $\underline{T}$ as an element in $\mathcal{C}_{\text {acf }}^{\Gamma \times \Gamma}(\underline{S})$ by doubling the $\Gamma$-action.

LEMmA 2.7.2. Let $\delta: \Gamma \rightarrow \mu_{n}$ be a character such that the push-forward $\delta_{*} T \in H^{1}\left(S, \mu_{n}\right)$ is trivial. Then for any $\psi \in \mathcal{C}_{\text {acf }}^{\Gamma}(\underline{S})$, we have the equality

$$
\vartheta_{\underline{S}}^{\delta}\left(\psi \times^{\Gamma} \underline{T}\right)=\vartheta_{\underline{S}}^{\delta}(\psi) .
$$

Proof. We use the observation that for any $\psi \in \mathcal{C}_{\text {acf }}^{\Gamma}(\underline{S})$, we have

$$
\vartheta_{\underline{S}}^{\delta}(\psi)=\vartheta_{\underline{S}}^{\text {id }}\left(\psi \times{ }^{\Gamma} \underline{\mu_{n}}\right),
$$

where on the right-hand side, $\Gamma$ acts on $\mu_{n}$ through $\delta$ and id: $\mu_{n} \rightarrow \mu_{n}$ is the identity. Indeed, by the definition of the isotypical component and (2.5.1), we see that both sides are equal to the $\widetilde{\delta}$-component of $\psi \times \mu_{n}$ with the product action of $\Gamma \times \mu_{n}$, where $\widetilde{\delta}$ is the character of $\Gamma \times \mu_{n}$ given by $\widetilde{\delta}(\gamma, \xi)=\delta(\gamma) \xi$. 


\section{Motivic Integration ON THE Hitchin FiBRATion}

Now by assumption, we have an isomorphism of $\mu_{n}$-torsors $T \times^{\Gamma} \mu_{n} \cong \mu_{n}$ and therefore

$$
\vartheta_{\underline{S}}^{\delta}\left(\psi \times{ }^{\Gamma} \underline{T}\right)=\vartheta_{\underline{S}}^{\text {id }}\left(\psi \times{ }^{\Gamma} \underline{T} \times{ }^{\Gamma} \underline{\mu_{n}}\right)=\vartheta_{\underline{S}}^{\text {id }}\left(\psi \times{ }^{\Gamma} \underline{\mu_{n}}\right)=\vartheta_{\underline{S}}^{\delta}(\psi) .
$$

\section{Orbifold volumes}

\subsection{Orbifold measure and stringy invariants}

We start with a well-known lemma.

Lemma 3.1.1. Consider a smooth separated $k \llbracket t \rrbracket$-scheme $X \rightarrow$ Spec $k \llbracket t \rrbracket$ of finite type, purely of relative dimension $d$. Let $\omega$ be a degree $d$ differential form on $X$ which generates the $k \llbracket t \rrbracket$-module of degree $d$ differential forms everywhere. Let $X_{k}$ denote the special fibre of $X$, and consider the definable morphism $\underline{\mathrm{ev}}: \underline{X}_{\circ} \rightarrow \underline{X_{k}}$ given by reducing modulo $t$. Then for every $\varphi \in \mathcal{C}_{\mathrm{acf}}\left(\underline{X_{k}}\right)$, we have

$$
\int_{\underline{X}_{\circ}} \underline{e v}^{*}(\varphi)|\omega|=\mathbb{L}^{-d} \int_{\underline{X_{k}}} \varphi
$$

in $\mathcal{C}_{\text {acf }}\left(*_{k}\right)$. The same statement holds with $\mathcal{C}_{\text {acf }}$ replaced by $\mathcal{C}_{\text {mot }}, \mathcal{C}_{\text {acf }}^{\Gamma}$ or $\mathcal{C}_{\text {mot }}^{\Gamma}$.

Proof. By the projection formula in Proposition 13.2.1(2) in [CL08], we have the equality

$$
\int_{\underline{X}_{\circ}} \underline{\mathrm{ev}}^{*}(\varphi)|\omega|=\int_{\underline{X_{k}}} \varphi \cdot \psi
$$

with $\psi$ the function $x \mapsto \int_{\underline{\mathrm{ev}}^{-1}(x)}|\omega|$. Since $X$ is smooth purely of relative dimension $d$ and $\omega$ is a $k \llbracket t \rrbracket$-generator everywhere, $\psi$ is constant equal to $\mathbb{L}^{-d}$.

We will now extend Lemma 3.1.1 to finite quotient singularities. For this, let $M$ be a smooth variety over $k \llbracket t \rrbracket$, purely of relative dimension $d$, and $\Gamma$ a finite abelian group acting generically freely on $M$. We assume that the $\Gamma$-orbit of every point is contained in an affine open subset, which is the case when $M$ is quasi-projective. Let $X=M / \Gamma$ denote the geometric quotient and $U \subset X$ the maximal open subvariety where the quotient morphism $\pi: M \rightarrow X$ is a $\Gamma$-torsor. We will always assume that $k$ contains a primitive root of unity $\xi$ of order $|\Gamma|$.

By abuse of notation, we write $I X$ for the coarse moduli space of the inertia stack of $[M / \Gamma]$. More explicitly, $I X$ can be identified with the disjoint union $I X=\bigsqcup_{\gamma \in \Gamma} M^{\gamma} / \Gamma$, where $M^{\gamma} \subset M$ denotes the fixed-point locus of $\gamma$. We will only be interested in the special fibre $I X_{k}=\sqcup_{\gamma \in \Gamma} M_{k}^{\gamma} / \Gamma$ of $I X$.

We define the definable subassignments $X^{\natural}, M^{\natural} \in$ GDef $_{\text {acf }, k}$ by

$$
X^{\natural}(\bar{K})=X(\bar{K} \llbracket t \rrbracket) \cap U(\bar{K}((t))) \quad \text { and } \quad M^{\natural}(\bar{K})=M(\bar{K} \llbracket t \rrbracket) \cap \pi^{-1} U(\bar{K}((t)))
$$

for any algebraically closed field $\bar{K}$ containing $k$.

We will always assume that $M$ admits a nowhere vanishing global $d$-form $\omega$ which is invariant under the action of $\Gamma$ (in particular, $\Gamma$ preserves the canonical bundle of $M$ ). Then $\omega$ descends to a $d$-form on $\omega_{\text {orb }}$ on $U$, which we then can integrate over $X^{\natural}$. To describe the resulting volume, we first construct a definable specialization morphism

$$
e: X^{\natural} \longrightarrow \underline{I X_{k}} .
$$

Construction 3.1.2 (Construction of $e$ ). Let $x \in\left|X^{\natural}\right|$, and set $K=k(x)$. Thus $x$ gives rise to a morphism

$$
x: \operatorname{Spec}(K \llbracket t \rrbracket) \longrightarrow X,
$$




\section{F. LOESER AND D. WYSS}

which maps the generic point to $U$. The fibre $T$ of $\pi: M \rightarrow X$ over $x_{\mid \operatorname{Spec}(K((t)))}$ is by construction a $\Gamma$-torsor. In particular, there exist a finite Galois extension $L / K((t))$ and a positive integer $r$ such that

$$
T=\operatorname{Spec}\left(L^{\times r}\right)
$$

with $L^{\times r}$ the product of $r$ copies of $L$. We write $T_{K \llbracket t \rrbracket}$ for the spectrum of the normalization of $K \llbracket t \rrbracket$ inside $L^{\times r}$; explicitly, we have $T_{K \llbracket t \rrbracket}=\operatorname{Spec}\left(K \llbracket t \rrbracket^{\times r}\right)$. We obtain a commutative diagram

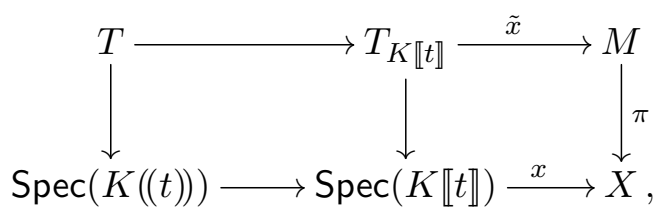

where we used the properness of $\pi$ to obtain the $\Gamma$-equivariant morphism $\tilde{x}$.

The inertia group $I \subset \operatorname{Gal}(L / K((t))) \subset \Gamma$ is naturally isomorphic to a subgroup of the group of roots of unity in $K$. Our choice of primitive root of unity $\xi$ thus gives a generator $\gamma$ of $I$. By the definition of the inertia group, $I$ acts trivially on the special fibre of $T_{K \llbracket t \rrbracket}$, and thus the image of $\tilde{x}_{\mid \operatorname{Spec}(K)}$ is a $K$-rational point of $M_{k}^{\gamma}$. Passing to the quotient, we obtain a well-defined $K$-point in $M_{k}^{\gamma} / \Gamma$, which we denote by $e(x)$.

Remark 3.1.3. The choice of $\xi$ gives, for every extension $K / k$, a splitting

$$
H^{1}(K((t)), \Gamma) \cong H^{1}(K, \Gamma) \oplus \Gamma .
$$

Torsors coming from $H^{1}(K, \Gamma)$ are called unramified, as they extend to $\operatorname{Spec}(K \llbracket t \rrbracket)$. Under this splitting, the class of the $\Gamma$-torsor $T$ appearing in Construction 3.1.2 is given by $\left[T_{K}, \gamma\right]$.

For any $\gamma \in \Gamma$, we will denote by $T_{\gamma}$ the $\Gamma$-torsor defined over $k$

$$
T_{\gamma}=\operatorname{Spec}\left(k\left(\left(t^{1 / r}\right)\right)\right) \times \operatorname{Spec}(k((t))) \Gamma /\langle\gamma\rangle,
$$

where $r$ denotes the order of $\gamma$ and $\gamma$ acts on $k\left(\left(t^{1 / r}\right)\right)$ through $\xi^{|\Gamma| / r}$. Its class is given by $[0, \gamma]$.

Proposition 3.1.4. The mapping $e:\left|X^{\natural}\right| \rightarrow\left|I X_{k}\right|$ is definable; that is, it is induced by a definable morphism $e: X^{\natural} \rightarrow \underline{I X_{k}}$.

Proof. It is enough to prove that for each $\gamma \in \Gamma$, the set $e^{-1}\left|M_{k}^{\gamma} / \Gamma\right|$ is definable, that is, of the form $\left|X^{\natural, \gamma}\right|$ with $X^{\natural, \gamma}$ a definable subset of $X^{\natural}$. Indeed, $X^{\natural}$ will then be the finite disjoint union of the sets $X^{\natural, \gamma}$, and the maps $X^{\natural, \gamma} \rightarrow M_{k}^{\gamma} / \Gamma$ are clearly definable. Since a point $x$ in $X^{\natural}$ belongs to $e^{-1}\left|M_{k}^{\gamma} / \Gamma\right|$ if and only its fibre $\overline{\pi^{-1}(x)}$ is isomorphic, as a definable $\Gamma$-set, to $T_{\gamma}$ over some finite extension $L$ of the field $k(x)$, the definability of $e^{-1}\left|M_{k}^{\gamma} / \Gamma\right|$ is a direct consequence of compactness in first-order logic. Indeed, consider a point $x$ in $e^{-1}\left|M_{k}^{\gamma} / \Gamma\right|$ and a finite extension $L$ of $k(x)$ such that $\pi^{-1}(x)$ is isomorphic, as a definable $\Gamma$-set, to $T_{\gamma}$ over $L$. Let $h$ be such an isomorphism. First, assume $L=k(x)$. Working in affine charts, $h$ can be expressed by a finite family of polynomials with coefficients in $k(x)$, say $N$ polynomials in at most $r$ variables and of degree at most $d$, and the existence of such an isomorphism $h$ can be expressed by a formula $\varphi_{x, \gamma}$ in the language $\mathcal{L}_{\mathrm{DP}, k(x)}$. It follows that there exists a formula $\psi_{x, \gamma}$ in the language $\mathcal{L}_{\mathrm{DP}, k}$ which is satisfied when one evaluates its free variables on the coordinates of $x$ and is such that if it is satisfied when evaluating the coordinates of some other point $y$, then there exists a definable isomorphism over $k(y)$ between $\pi^{-1}(y)$ and $T_{\gamma}$. In general, fix a positive integer $m$. One can encode field extensions $L$ of degree $m$ of a perfect field $E$ through 


\section{Motivic INTEGRATion ON THE Hitchin FibRATion}

the minimal polynomial of a generator, as explained in Section 3.2 of [CHL11]. In particular, the existence of a field extension of degree $m$ can be expressed as the existence of an $m$-tuple $\left(a_{1}, \ldots, a_{m}\right)$ satisfying the definable condition expressing the irreducibility of the corresponding monic polynomial $P=x^{m}+a_{1} x^{m-1}+\cdots+a_{m}$, and working in the basis $\left(1, x, \ldots, x^{m-1}\right)$ of $L=E[x] / P$ allows one to express definable conditions involving polynomials with coefficients in $L$ as definable conditions on tuples of elements of $E$. In our case, the existence of an isomorphism over $L$ may thus be rephrased as a definable condition on tuples of elements of $k(x)$. Thus, there exists a formula $\psi_{x, \gamma, m}$ in the language $\mathcal{L}_{\mathrm{DP}, k}$ which is satisfied when one evaluates its free variables on the coordinates of $x$ and is such that, furthermore, for any point $y$ in the given affine open set, if it is satisfied when evaluating the coordinates of $y$, then there exist an extension $L$ of degree $m$ of $k(y)$ and a definable isomorphism over $L$ between $\pi^{-1}(y)$ and $T_{\gamma}$. The set of all points $y$ satisfying $\psi_{x, \gamma, m}$ is an $\mathcal{L}_{\mathrm{DP}, k}$-definable subset $Z_{\psi_{x, \gamma, m}}$ of $X^{\natural}$. By construction, $X^{\natural}$ is covered by the set of all such definable sets $Z_{\psi_{x, \gamma, m}}$. Thus, by compactness, $X^{\natural}$ is the union of finitely many such definable sets, say $Z_{\psi_{x_{i}, \gamma_{i}, m_{i}}}$. For each $\gamma$, define $X^{\natural, \gamma}$ as the union of the sets $Z_{\psi_{x_{i}, \gamma_{i}, m_{i}}}$ with $\gamma_{i}=\gamma$. It is a definable set, and $\left|X^{\mathrm{h}, \gamma}\right|=e^{-1}\left|M_{k}^{\gamma} / \Gamma\right|$.

Definition 3.1.5 (Weight function). For any point $x \in M_{k}^{\gamma}$, consider the action of $\gamma$ on the tangent space $T_{x} M_{k}$. There are unique positive integers $1 \leqslant c_{1}, \ldots, c_{d} \leqslant|\Gamma|$ such that the eigenvalues of $\gamma$ are given by $\xi^{c_{1}}, \ldots, \xi^{c_{d}}$. We define the weight $w_{x}(\gamma)$ as

$$
w_{x}(\gamma)=\frac{1}{|\Gamma|} \sum_{i=1}^{d} c_{i}
$$

Clearly, $w_{x}(\gamma)$ is locally constant as a function in $x$. For simplicity, we will further assume that $w_{x}(\gamma)$ is constant on the components of $M_{k}^{\gamma}$ and write $w(\gamma)$ for its value on $\mathrm{M}_{k}^{\gamma}$. Also notice that, by assumption, $\Gamma$ preserves the canonical bundle of $M$ and hence $w(\gamma) \in \mathbb{Z}$.

In our case, we are interested in integrating the twists $M^{\natural} \times{ }^{\Gamma} T_{\gamma^{-1}}$ over $X^{\natural}$. Here by $M^{\natural} \times^{\Gamma} T_{\gamma^{-1}}$, we of course mean the image of $M^{\natural} \times \underline{T_{\gamma^{-1}}}$ in $M \times{ }^{\Gamma} T_{\gamma^{-1}}$.

By the construction of the morphism $e$ in Construction 3.1.2 and Remark 3.1.3, we see that $M^{\natural} \times^{\Gamma} T_{\gamma^{-1}}$ is supported on

$$
X^{\natural, \gamma}:=e^{-1} \underline{\left(M_{k}^{\gamma} / \Gamma\right)} \subset X^{\natural} .
$$

Theorem 3.1.6. For every $\gamma \in \Gamma$, we have

$$
\int_{X^{\natural, \gamma}}^{\text {mot }} M^{\natural} \times^{\Gamma} T_{\gamma^{-1}}\left|\omega_{\text {orb }}\right|=\mathbb{L}^{-w(\gamma)}\left[M_{k}^{\gamma}\right]
$$

in $\mathcal{C}_{\text {mot }}^{\Gamma}\left(*_{k}\right)$. In particular, for any $\varrho \in \widehat{\Gamma}$,

$$
\int_{X^{\natural, \gamma}}^{\text {mot, } \varrho} M^{\natural} \times{ }^{\Gamma} T_{\gamma^{-1}}\left|\omega_{\text {orb }}\right|=\mathbb{L}^{-w(\gamma)} \vartheta_{*_{k}}^{\varrho}\left(\left[M_{k}^{\gamma}\right]\right)
$$

in $\mathcal{C}_{\text {mot }}\left(*_{k}\right)$.

\subsection{Proof of Theorem 3.1.6}

We start by proving a local version of Theorem 3.1.6. 


\section{F. LOESER AND D. WYSS}

Proposition 3.2.1. Assume that $\Gamma$ acts linearly on $M=\mathbb{A}_{k \llbracket t \rrbracket}^{d}$, and fix a $\gamma \in \Gamma$. Let 0 denote the image of the origin in $\left(\mathbb{A}_{k}^{d}\right)^{\gamma} / \Gamma \subset I M_{k}$. We have the relation

$$
\int_{e^{-1}(0)}^{\operatorname{mot}} M^{\natural} \times^{\Gamma} T_{\gamma^{-1}}\left|\omega_{\text {orb }}\right|=\mathbb{L}^{-w(\gamma)}
$$

in $\mathcal{C}_{\text {mot }}^{\Gamma}\left(*_{k}\right)$, where $\mathbb{L}$ is considered with the trivial $\Gamma$-action. Moreover, for any $\delta \in \widehat{\Gamma}$ which is non-trivial,

$$
\int_{e^{-1}(0)}^{\text {mot }, \delta} M^{\natural} \times^{\Gamma} T_{\gamma^{-1}}\left|\omega_{\text {orb }}\right|=0 .
$$

Proof. Let $I \subset \Gamma$ be the cyclic group generated by $\gamma$ and $r=|I|$. We may assume that $\Gamma$ acts diagonally on $\mathbb{A}_{k \llbracket t \rrbracket}^{d}$ and that $\gamma$ has eigenvalues $\xi^{c_{1}}, \ldots, \xi^{c_{d}}$ for unique integers $1 \leqslant c_{i} \leqslant d$. We then use (3.1.2) to get the description

$$
\mathbb{A}_{k((t))}^{d} \times^{\Gamma} T_{\gamma^{-1}} \cong \operatorname{Spec}\left(k\left(\left(t^{1 / r}\right)\right)\left[x_{1}, \ldots, x_{n}\right]^{I}\right),
$$

where $\gamma$ acts on $k\left(\left(t^{1 / r}\right)\right)$ by $\gamma \cdot t^{1 / r}=\xi t^{1 / r}$. We then define a $k((t))$-morphism

$$
\lambda_{\gamma}: \mathbb{A}_{k((t))}^{d} \longrightarrow \mathbb{A}_{k((t))}^{d} \times{ }^{\Gamma} T_{\gamma^{-1}}
$$

on the level of coordinate rings by $f\left(x_{d}, \ldots, x_{d}\right) \mapsto f\left(t^{c_{1} / r} x_{1}, \ldots, t^{c_{n} / r} x_{n}\right)$. As in [DL02, § 2.3], one sees that $\lambda_{\gamma}$ is indeed defined over $k((t))$, and since $t$ is invertible, it is in fact an isomorphism. We thus obtain a cartesian square

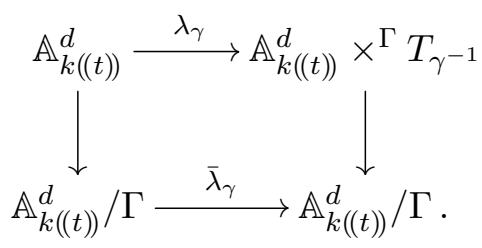

Now let $Y$ be the image of $\underline{M}$ in $\underline{M / \Gamma}$. It is a definable subassignment of $\underline{M / \Gamma}$. Set $Y^{\natural}=$ $Y \cap\left(\mathbb{A}^{d} / \Gamma\right)^{\natural}$. Then by [DL02, $\left.\S 2.7\right]$, we have a definable bijection $\bar{\lambda}_{\gamma}: Y^{\natural} \rightarrow e^{-1}(0)$, and hence

$$
\int_{e^{-1}(0)}^{\text {mot }} M^{\natural} \times^{\Gamma} T_{\gamma^{-1}}\left|\omega_{\text {orb }}\right|=\int_{Y^{\natural}}^{\text {mot }} M^{\natural}\left|\bar{\lambda}_{\gamma}^{*} \omega_{\text {orb }}\right| .
$$

By the definition of $\bar{\lambda}_{\gamma}$, we have

$$
\bar{\lambda}_{\gamma}^{*} \omega_{\text {orb }}=t^{\sum_{i} c_{i} / r} \omega_{\text {orb }}=t^{w(\gamma)} \omega_{\text {orb }}
$$

Hence

$$
\int_{Y^{\natural}}^{\text {mot }} M^{\natural}\left|\bar{\lambda}_{\gamma}^{*} \omega_{\text {orb }}\right|=\int_{Y^{\natural}}^{\text {mot }} M^{\natural}\left|t^{w(\gamma)} \omega_{\text {orb }}\right|=\mathbb{L}^{-w(\gamma)} \int_{Y^{\natural}}^{\text {mot }} M^{\natural}\left|\omega_{\text {orb }}\right| .
$$

So it remains to prove that

$$
\int_{Y^{\natural}}^{\text {mot }} M^{\natural}\left|\omega_{\text {orb }}\right|=1
$$

Let $M_{k}^{\circ}$ be the complement in $M_{k}$ of the coordinate hyperplanes. Consider the morphism ac $\underline{M / \Gamma} \rightarrow \underline{M_{k} / \Gamma}$. We set $Y^{\sharp}=Y^{\natural} \cap\left(\operatorname{ac}^{-1}\left(\underline{M_{k}^{\circ} / \Gamma}\right)\right)$ and $M^{\sharp}=M^{\natural} \cap\left(\operatorname{ac}^{-1}\left(\underline{M_{k}^{\circ}}\right)\right)$. Since 


\section{Motivic INTEGRATion ON THE Hitchin FibRATion}

$\operatorname{ac}^{-1}\left(\underline{M_{k}} \backslash M_{k}^{\circ}\right)$ is of dimension less than $d$,

$$
\int_{Y^{\natural}}^{\text {mot }} M^{\natural}\left|\omega_{\text {orb }}\right|=\int_{Y^{\sharp}}^{\text {mot }} M^{\sharp}\left|\omega_{\text {orb }}\right| .
$$

Note that $M^{\sharp}$ is definably isomorphic to $\left\{(y, x) \in Y^{\sharp} \times \underline{M_{k}} \mid\right.$ ac $\left.(y)=p(x)\right\}$, with $p$ the projection $M_{k}^{\circ} \rightarrow M_{k}^{\circ} / \Gamma$. Indeed, any point $z$ in $M^{\sharp}$ is uniquely determined by its image in $Y^{\sharp}$ and ac $(z)$.

Thus, if $\varphi$ denotes the class of $p: M_{k}^{\circ} \rightarrow M_{k}^{\circ} / \Gamma$ in $\mathcal{C}_{\text {mot }}^{\Gamma}\left(\underline{M_{k}^{\circ} / \Gamma}\right)$,

$$
\int_{Y^{\sharp}}^{\mathrm{mot}} M^{\sharp}\left|\omega_{\text {orb }}\right|=\int_{Y^{\sharp}}^{\mathrm{mot}} \operatorname{ac}^{*}(\varphi)\left|\omega_{\text {orb }}\right| .
$$

Now let $y \in \operatorname{ac}\left(Y^{\sharp}\right)$ and $x \in M_{k}^{\circ}$ with $p(x)=y$. We have

$$
\int_{\mathrm{ac}^{-1}(y) \cap Y^{\sharp}}^{\text {mot }}\left|\omega_{\mathrm{orb}}\right|=\int_{\mathrm{ac}^{-1}(x) \cap M^{\sharp}}^{\mathrm{mot}}\left|\omega_{M}\right|=\int_{\mathrm{ac}^{-1}(x)}^{\mathrm{mot}}\left|\omega_{M}\right|=(\mathbb{L}-1)^{-d} .
$$

By Proposition 2.4.3 (Fubini theorem), we deduce that

$$
\int_{Y^{\sharp}}^{\mathrm{mot}} \operatorname{ac}^{*}(\varphi)\left|\omega_{\text {orb }}\right|=(\mathbb{L}-1)^{-d} \int_{\underline{M_{k}^{\circ} / \Gamma}}^{\mathrm{mot}}\left[M_{k}^{\circ}\right] .
$$

So, to conclude, it is enough to verify that the class of $M_{k}^{\circ}$ in $\mathcal{C}_{\text {mot }}^{\Gamma}\left(*_{k}\right)$ is equal to $(\mathbb{L}-1)^{d}$, with trivial $\Gamma$-action. This follows from Lemma 2.5.2 for the isogeny $M_{k}^{\circ} \rightarrow M_{k}^{\circ} / \Gamma$ since $M_{k}^{\circ} \cong \mathbb{G}_{m, k}^{d}$.

Next we go back to the situation of Theorem 3.1.6 but considering only a global fixed point. Proposition 3.2.2. Let $x \in\left|M_{k}\right|$ be fixed by $\Gamma$. Then for any $\gamma \in \Gamma$, we have

$$
\int_{e^{-1}(x)}^{\text {mot }} M^{\natural} \times^{\Gamma} T_{\gamma^{-1}}\left|\omega_{\text {orb }}\right|=\mathbb{L}^{-w(\gamma)} \in \mathcal{C}_{\text {mot }}^{\Gamma}\left(*_{k(x)}\right) .
$$

In particular, for any $\delta \in \widehat{\Gamma}$ which is non-trivial,

$$
\int_{e^{-1}(x)}^{\text {mot, } \delta} M^{\natural} \times^{\Gamma} T_{\gamma^{-1}}\left|\omega_{\text {orb }}\right|=0 .
$$

Proof. After base change, we may assume that $x$ is a $k$-rational point. By [GWZ20, Lemma 4.14], there exist a $\Gamma$-invariant open neighbourhood $U \subset M$ of $x$ and an étale morphism $f: U \rightarrow \mathbb{A}_{k \llbracket t \rrbracket}^{d}$ which is $\Gamma$-invariant for some linear action of $\Gamma$ on $\mathbb{A}_{k \llbracket t \rrbracket}^{d}$ and sends $x$ to the origin (in [GWZ20], the lemma is only proven for non-archimedean local fields, but the proof applies to any Henselian ring). Furthermore, $f$ induces a definable bijection

$$
\bar{f}: e^{-1}(x) \longrightarrow e_{\mathbb{A}_{k \llbracket t \rrbracket}^{d}}^{-1}(0) .
$$

Since $f$ is étale, we have $\left|f^{*} \omega_{\text {orb, } \mathbb{A}_{k \llbracket t !}^{n} \mid}\right|=\left|\omega_{\text {orb }, M}\right|$, and thus the proposition follows from Proposition 3.2.1.

Finally, we can prove the general pointwise statement.

Proposition 3.2.3. For any point $x \in\left|M_{k}^{\gamma} / \Gamma\right|$, we have

$$
\int_{e^{-1}(x)}^{\text {mot }} M^{\natural} \times^{\Gamma} T_{\gamma^{-1}}\left|\omega_{\text {orb }}\right|=\mathbb{L}^{-w(\gamma)}\left[\pi^{-1}(x)\right]
$$




\section{F. LOESER AND D. WYSS}

in $\mathcal{C}_{\text {mot }}^{\Gamma}\left(*_{k(x)}\right)$, where $\pi: M_{k}^{\gamma} \rightarrow M_{k}^{\gamma} / \Gamma$ denotes the quotient morphism. In particular, for any $\delta \in \widehat{\Gamma}$,

in $\mathcal{C}_{\text {mot }}\left(*_{k(x)}\right)$.

$$
\int_{e^{-1}(x)}^{\text {mot, } \delta} M^{\natural} \times^{\Gamma} T_{\gamma^{-1}}\left|\omega_{\text {orb }}\right|=\mathbb{L}^{-w(\gamma)} \vartheta_{*_{k(x)}}^{\delta}\left(\left[\pi^{-1}(x)\right]\right)
$$

Proof. After base change, we may assume that $x$ is a $k$-rational point. Let $\Gamma^{\prime} \subset \Gamma$ denote the stabilizer group of $x$. We denote by $e_{\Gamma^{\prime}}$ and $\omega_{\mathrm{orb}, \Gamma^{\prime}}$ the specialization morphism and orbifold form for $M / \Gamma^{\prime}$. We have a commutative diagram

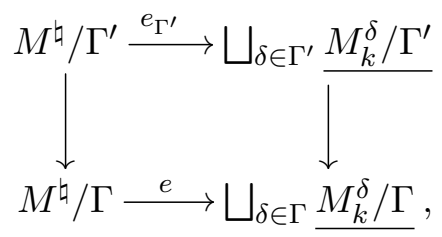

where the vertical arrows are quotient morphisms.

We first assume that $x$ lifts to a $k$-rational point $\tilde{x} \in M_{k}^{\gamma}$, in which case we have $\pi^{-1}(x) \cong \Gamma / \Gamma^{\prime}$. Then we have an identification

$$
e_{\Gamma^{\prime}}^{-1}\left(\pi^{-1}(x)\right)=\bigsqcup_{x^{\prime} \in \pi^{-1}(x)} e_{\Gamma^{\prime}}^{-1}\left(x^{\prime}\right)
$$

and the free $\Gamma / \Gamma^{\prime}$-action on $e_{\Gamma^{\prime}}^{-1}\left(\pi^{-1}(x)\right)$ simply permutes the components on the right-hand side. Therefore, we have an injective morphism

$$
\sigma: e_{\Gamma^{\prime}}^{-1}(\tilde{x}) \longrightarrow \bigsqcup_{x^{\prime} \in \pi^{-1}(x)} e_{\Gamma^{\prime}}^{-1}\left(x^{\prime}\right) \longrightarrow e^{-1}(x)
$$

which is in fact an isomorphism. Indeed, for any algebraically closed field extension $K / k$, any $K$-point $y: \operatorname{Spec}(K((t))) \rightarrow M / \Gamma$ in $e^{-1}(x)$ lifts by the definition of a $\Gamma$-equivariant morphism $T_{\gamma} \rightarrow M$. As $\gamma \in \Gamma^{\prime}$, the quotient $T_{\gamma} / \Gamma^{\prime}$ is the trivial $\Gamma / \Gamma^{\prime}$-torsor, which gives a lift of $y$ to $e_{\Gamma^{\prime}}^{-1}(\tilde{x})$.

From the isomorphism $M^{\natural} \times M^{\natural} / \Gamma M^{\natural} \cong M^{\natural} \times \Gamma$, we see that

$$
\left(M^{\natural} \times{ }^{\Gamma} T_{\gamma^{-1}}\right) \times_{M^{\natural} / \Gamma} M^{\natural} / \Gamma^{\prime} \cong\left(M^{\natural} \times{ }^{\Gamma} T_{\gamma^{-1}}\right) \times{ }^{\Gamma^{\prime}} \Gamma .
$$

Hence, we have

$$
\begin{aligned}
\int_{e^{-1}(x)}^{\text {mot }} M^{\natural} \times^{\Gamma} T_{\gamma^{-1}}\left|\omega_{\text {orb }}\right| & =\int_{e_{\Gamma^{\prime}}^{-1}(\tilde{x})}^{\text {mot }} \sigma^{*}\left(M^{\natural} \times{ }^{\Gamma} T_{\gamma^{-1}}\right)\left|\sigma^{*} \omega_{\text {orb }}\right| \\
& =\int_{e_{\Gamma^{\prime}}^{-1}(\tilde{x})}^{\operatorname{mot}}\left(M^{\natural} \times^{\Gamma} T_{\gamma^{-1}}\right) \times \Gamma^{\prime} \Gamma\left|\omega_{\text {orb }, \Gamma^{\prime}}\right|=\mathbb{L}^{-w(\gamma)}\left[\Gamma / \Gamma^{\prime}\right] .
\end{aligned}
$$

For the last equation, we used Lemma 2.7.1 and Proposition 3.2.2.

If $x \in M^{\gamma} / \Gamma(k)$ does not lift to a $k$-point of $M^{\gamma}$, we can find a $\Gamma$-torsor $T$ over $\operatorname{Spec}(k)$ such that $x$ lifts to a $k$-point $\tilde{x}$ in $M^{\gamma} \times^{\Gamma} T$, in which case we have $\pi^{-1}(x) \cong \Gamma / \operatorname{Stab}(\tilde{x}) \times^{\Gamma} T^{-1}$. We can consider $T$ as an unramified $k \llbracket t \rrbracket$-torsor and use Lemma 2.7.1 to write

$$
\int_{e^{-1}(x)}^{\text {mot }} M^{\natural} \times^{\Gamma} T_{\gamma^{-1}}\left|\omega_{\text {orb }}\right|=\left(\int_{e^{-1}(x)}^{\text {mot }}\left(M^{\natural} \times^{\Gamma} T\right) \times^{\Gamma} T_{\gamma^{-1}}\left|\omega_{\text {orb }}\right|\right) \times \times^{\Gamma} T^{-1} .
$$

Repeating the previous argument with $M^{\natural}$ replaced by $M^{\natural} \times^{\Gamma} T$ then proves the proposition. 


\section{Motivic Integration ON THE Hitchin FibRATion}

Proof of Theorem 3.1.6. We can see the equality

$$
\int_{e^{-1}(\gamma)} M^{\natural} \times{ }^{\Gamma} T_{\gamma^{-1}}\left|\omega_{\text {orb }}\right|=\mathbb{L}^{-w(\gamma)}\left[M^{\gamma}\right]
$$

as the push-forward to the point of an equality in $\mathcal{C}_{\text {mot }}^{\Gamma}\left(M^{\gamma} / \Gamma\right)$. The equality can thus be checked pointwise for every $x \in\left|M^{\gamma} / \Gamma\right|$, which is exactly Proposition 3.2.3.

\subsection{E-polynomials and stringy invariants}

In this subsection, let $k=\mathbb{C}$.

3.3.1 E-polynomials of varieties. Recall, for example from [BD96], that one has a realization homomorphism

$$
E: K_{0}\left(\operatorname{Var}_{k}\right) \longrightarrow \mathbb{Z}[x, y]
$$

given by the $E$-polynomial. For an algebraic variety $M$ over $k$, it is defined by the Hodge-Deligne polynomial

$$
E(M ; x, y)=\sum_{i, p, q \geqslant 0}(-1)^{i} h_{c}^{i ; p, q}(X) x^{p} y^{q},
$$

where the $h_{c}^{i ; p, q}(M)$ are the compactly supported mixed Hodge numbers of $M$.

By the functoriality of Deligne's mixed Hodge structures [Del71], one can do the same construction equivariantly. This way, for a finite abelian group $\Gamma$, one obtains a morphism

$$
E^{\Gamma}: K_{0}\left(\operatorname{Var}_{k}^{\Gamma}\right) \longrightarrow R_{\Gamma}[x, y],
$$

where $R_{\Gamma}$ denotes the (complex) representation ring of $\Gamma$, that is, the group ring of the character group $\widehat{\Gamma}$. Furthermore, $E^{\Gamma}$ admits a decomposition into isotypical components

$$
E^{\Gamma}=\bigoplus_{\varrho \in \widehat{\Gamma}} E^{\varrho} .
$$

Note, however, that for $\varrho \not \equiv 1$, the component $E^{\varrho}$ is only additive and not multiplicative.

Next we recall the definition of the stringy $E$-polynomial. We restrict ourselves to the special case where $M$ is a smooth $k$-variety with an action of a finite abelian group $\Gamma$ preserving the canonical bundle of $M$. As before, we also assume that the weight function $w$ is constant on $M^{\gamma}$ for each $\gamma \in \Gamma$. Then we define the stringy $E$-polynomial of the quotient stack $\mathbb{X}=[M / \Gamma]$ by

$$
E_{\mathrm{st}}(\mathbb{X} ; x, y)=\sum_{\gamma \in \Gamma}(x y)^{\operatorname{dim} M-w(\gamma)} E\left(M^{\gamma} / \Gamma ; x, y\right) .
$$

We will also need a twisted version $E_{\mathrm{st}}^{\varrho}$ of $E_{\text {st }}$ for a class $\varrho \in H_{\mathrm{grp}}^{2}\left(\Gamma, \mu_{n}\right)$, where $H_{\mathrm{grp}}^{2}$ denotes group cohomology. Such a class defines, for every $\gamma \in \Gamma$, a character $\varrho_{\gamma}: \Gamma \rightarrow \mu_{n}$, and we set

$$
E_{\mathrm{st}}^{\varrho}(\mathbb{X} ; x, y)=\sum_{\gamma \in \Gamma}(x y)^{\operatorname{dim} M-w(\gamma)} E^{\varrho_{\gamma}}\left(M^{\gamma} ; x, y\right) .
$$

Remark 3.3.2. The notation $E_{\mathrm{st}}^{\varrho}(\mathbb{X} ; x, y)$ is slightly misleading as the polynomial depends not only on $\mathbb{X}$ but rather on $M$ as a $\Gamma$-variety.

3.3.3 E-polynomials of Chow motives. The E-polynomial factors through the Grothendieck ring of Chow motives. More precisely, there exists a Hodge realization functor

$$
\operatorname{DM}_{\mathrm{gm}}(k, \mathbb{Q}) \longrightarrow D^{b}\left(\mathrm{MHS}_{\mathbb{Q}}^{p}\right)
$$




\section{F. LOESER AND D. WYSS}

with values in the bounded derived category of polarized mixed $\mathbb{Q}$-Hodge structures, cf. [Lev98, Hub00], which extends to a functor

$$
\operatorname{DM}_{\mathrm{gm}}(k, \Lambda) \longrightarrow D^{b}\left(\mathrm{MHS}_{\mathbb{C}}^{p}\right)
$$

with values in the bounded derived category of polarized mixed $\mathbb{C}$-Hodge structures. Since, as recalled in Remark 2.1.2, the ring $K_{0}\left(\mathrm{DM}_{\mathrm{gm}}(k, \Lambda)\right)$ is isomorphic to $K_{0}\left(\mathrm{M}_{\text {rat }}(k, \Lambda)\right)$, it follows that the $E$-polynomial factors through a morphism

$$
E: K_{0}\left(\mathrm{M}_{\mathrm{rat}}(k, \Lambda)\right) \longrightarrow \mathbb{Z}[x, y] .
$$

Now if $X$ is a reduced and separated $k$-scheme of finite type endowed with an action of a finite abelian group $\Gamma$ and $\varrho$ is a complex character of $\Gamma$, we have that $E^{\varrho}([X])=E\left(\chi_{*, c}^{\varrho}(X)\right)$, using notation from Section 2.4.

\section{Néron models, isogenies and pairings}

\subsection{Néron models}

Let $k$ be a field of characteristic zero and $\mathcal{P}$ an abelian variety over $k((t))$. The Néron model $\mathcal{N}(\mathcal{P})$ of $\mathcal{P}$ is the unique smooth, separated and finite-type group scheme over $k \llbracket t \rrbracket$ such that for any smooth $k \llbracket t \rrbracket$-scheme $X$ and any morphism $X_{k(t))} \rightarrow \mathcal{P}$, there exists a unique extension $X \rightarrow \mathcal{N}(\mathcal{P})$. In particular, for each extension $K / k$, we have a bijection

$$
\mathcal{P}(K((t))) \stackrel{\sim}{\longrightarrow} \mathcal{N}(\mathcal{P})(K \llbracket t \rrbracket) .
$$

This implies that the assignments $\underline{\mathcal{P}}$ and $\underline{\mathcal{N}(\mathcal{P})}$ 。 are isomorphic, which is why Néron models appear naturally in the context of motivic integration, as first observed in [LS03]. The following remark makes this more concrete.

Remark 4.1.1. Since the canonical bundle of $\mathcal{P}$ is trivial and $\mathcal{P}$ is proper, we can choose a global translation-invariant volume form $\omega$ on $\mathcal{P}$, which is unique up to a scalar in $k((t))$. If $\operatorname{ord}_{\mathcal{N}} \omega \in \mathbb{Z}$ denotes the order of vanishing of $\omega$ along the special fibre $\mathcal{N}(\mathcal{P})$, then $t^{-\operatorname{ord}_{\mathcal{N}} \omega} \cdot \omega$ is a global volume form on $\mathcal{N}(\mathcal{P})$. By Lemma 3.1.1 together with Proposition 12.6 of [CL15], we have

$$
\int_{\underline{\mathcal{P}}}|\omega|=\mathbb{L}^{-\operatorname{ord}_{\mathcal{N}} \omega} \int_{\underline{\mathcal{N}(\mathcal{P})}}\left|t^{-\operatorname{ord}_{\mathcal{N}} \omega} \cdot \omega\right|=\mathbb{L}^{-d-\operatorname{ord}_{\mathcal{N}} \omega}\left[\mathcal{N}(\mathcal{P})_{k}\right],
$$

with $d$ the dimension of $\mathcal{P}$. Indeed, Lemma 3.1.1 implies the second equality, while Proposition 12.6 of [CL15] implies the equality between the left-hand side of the first equation and the right-hand side of the last equation.

Let $\mathcal{N}^{0}(\mathcal{P})$ denote the fibrewise identity component of $\mathcal{N}(\mathcal{P})$. The quotient

$$
\Phi_{\mathcal{P}}=\frac{\mathcal{N}(\mathcal{P})_{k}}{\mathcal{N}^{0}(\mathcal{P})_{k}}
$$

is a finite étale $k$-group scheme called the component group of $\mathcal{N}(\mathcal{P})$. If $k$ is algebraically closed, we will consider $\Phi_{\mathcal{P}}$ simply as a finite group.

As for finite groups in Remark 3.1.3, we call a $\mathcal{P}$-torsor $T$ unramified if $T$ becomes trivial over $\bar{k}((t))$, that is, if $T(\bar{k}((t))) \neq \emptyset$. It then follows from [BLR90, Corollary 6.5.3] that $T$ extends uniquely to a $\mathcal{N}(\mathcal{P})$-torsor over $k \llbracket t \rrbracket$ which we denote by $\mathcal{N}(T)$. 


\section{Motivic INTEGRATion ON THE Hitchin FibRATion}

\subsection{Self-dual isogenies}

In this subsection, we assume $k$ to be algebraically closed of characteristic zero. Let $\mathcal{P}$ and $\mathcal{P}^{\prime}$ be abelian varieties over $k((t))$ and

$$
\phi: \mathcal{P} \longrightarrow \mathcal{P}^{\prime}
$$

be an isogeny with kernel $\Gamma$. By [BLR90, Proposition 7.3.6], the map $\phi$ extends to an isogeny of Néron models $\mathcal{N}(\phi): \mathcal{N}(\mathcal{P}) \rightarrow \mathcal{N}\left(\mathcal{P}^{\prime}\right)$, meaning that $\mathcal{N}(\phi)$ is fibrewise finite and surjective on identity components. The kernel $\underline{\Gamma}=\operatorname{ker}(\mathcal{N}(\phi))$ is an étale group scheme over $k \llbracket t \rrbracket$; in particular, we have an identification of finite groups

$$
\Gamma(k((t)))=\underline{\Gamma}(k \llbracket t \rrbracket)=\underline{\Gamma}_{k}(k) .
$$

Notice, however, that in general $\Gamma(\overline{k((t))}) \neq \Gamma(k((t)))$ as $\mathcal{N}(\phi)$ is only quasi-finite. The following two finite groups will be interesting for us later:

$$
\Gamma^{0}=\underline{\Gamma}(k \llbracket t \rrbracket) \cap \mathcal{N}^{0}(\mathcal{P})(k \llbracket t \rrbracket), \quad \Gamma^{\prime}=\underline{\Gamma}(k \llbracket t \rrbracket) / \Gamma^{0} .
$$

The short exact sequence of abelian group schemes over $k((t))$

$$
0 \longrightarrow \Gamma \longrightarrow \mathcal{P} \longrightarrow \mathcal{P}^{\prime} \longrightarrow 0
$$

gives rise to a long exact sequence of étale cohomology groups

$$
0 \longrightarrow \Gamma(k((t))) \longrightarrow \mathcal{P}(k((t))) \stackrel{\phi}{\longrightarrow} \mathcal{P}^{\prime}(k((t))) \stackrel{\partial_{\phi}}{\longrightarrow} H^{1}(k((t)), \Gamma) \longrightarrow H^{1}(k((t)), \mathcal{P}) \longrightarrow \cdots
$$

The image of $\partial_{\phi}$ admits a description in terms of Néron models by means of the following commutative diagram with exact rows:

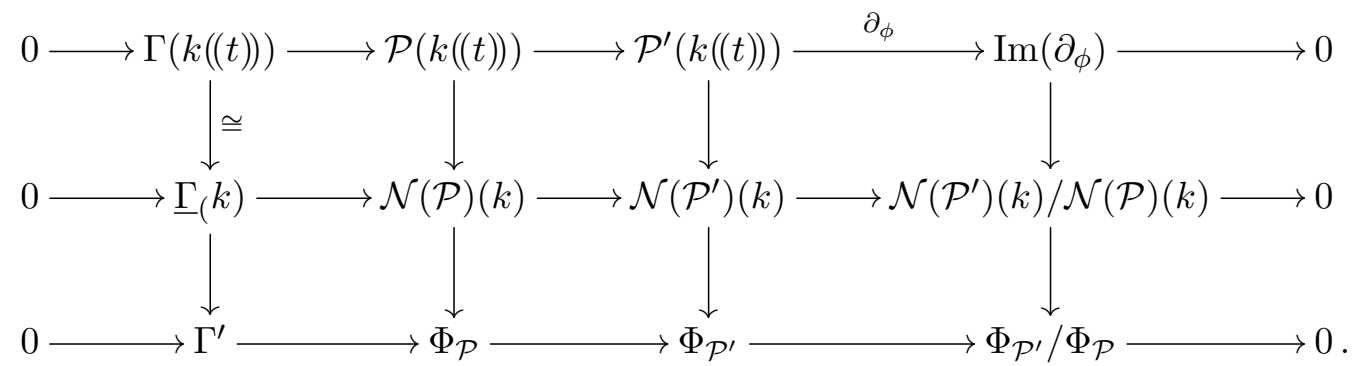

Here we write $\mathcal{N}(\mathcal{P})(k)$ for $\mathcal{N}(\mathcal{P})_{k}(k)$ and $\mathcal{P}(k((t))) \rightarrow \mathcal{N}(\mathcal{P})(k)$ for the composition $\mathcal{P}(k((t))) \cong$ $\mathcal{N}(\mathcal{P})(k \llbracket t \rrbracket) \rightarrow \mathcal{N}(\mathcal{P})(k)$, and similar for $\mathcal{P}^{\prime}$. We claim that both arrows in the composition

$$
\operatorname{Im}\left(\partial_{\phi}\right) \longrightarrow \mathcal{N}\left(\mathcal{P}^{\prime}\right)(k) / \mathcal{N}(\mathcal{P})(k) \longrightarrow \Phi_{\mathcal{P}} / \Phi_{\mathcal{P}^{\prime}}
$$

are isomorphisms. Indeed, for the first arrow we notice that

$$
\operatorname{ker}(\mathcal{P}(k((t))) \longrightarrow \mathcal{P}(k)) \cong \operatorname{ker}\left(\mathcal{P}^{\prime}(k((t))) \longrightarrow \mathcal{P}^{\prime}(k)\right)
$$

as the restriction $\mathcal{P}(k((t))) \rightarrow \mathcal{P}(k)$ induces a bijection on torsion points [BLR90, Proposition 7.3.3]. The second arrow is an isomorphism since the map $\mathcal{N}^{0}(\mathcal{P})(k) \rightarrow \mathcal{N}^{0}\left(\mathcal{P}^{\prime}\right)(k)$ is surjective.

Lemma 4.2.1. Let $m=\left|\Phi_{\mathcal{P}}\right|, m^{\prime}=\left|\Phi_{\mathcal{P}^{\prime}}\right|$ and $\phi^{r}$ be the composition $\phi \circ[r]$ for any positive integer $r$. Then we have

$$
\operatorname{Im}\left(\partial_{\phi}\right)=\operatorname{ker}\left(H^{1}(k((t)), \Gamma) \longrightarrow H^{1}\left(k((t)), \operatorname{ker}\left(\phi^{m^{\prime}}\right)\right)\right)
$$

and

$$
\Gamma^{0}=\operatorname{Im}\left(\operatorname{ker}\left(\phi^{m}\right)(k((t))) \stackrel{[m]}{\longrightarrow} \mathcal{P}[n](k((t)))\right) .
$$




\section{F. LOESER AND D. WYSS}

Proof. Consider the commuting diagram

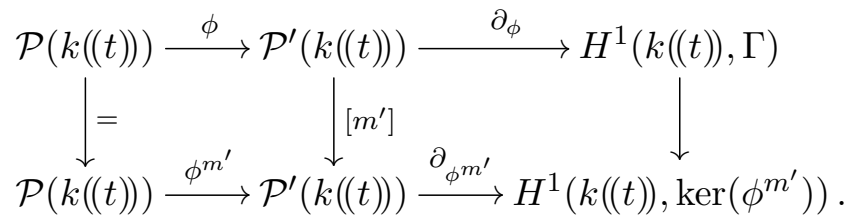

By construction, $\operatorname{Im}\left(\left[m^{\prime}\right]\right) \subset \mathcal{N}^{0}\left(\mathcal{P}^{\prime}\right)(k \llbracket t \rrbracket)$, and since $\mathcal{N}^{0}\left(\mathcal{P}^{\prime}\right)(k \llbracket t \rrbracket)$ is divisible, we deduce $\partial_{\phi^{m^{\prime}}} \circ$ $\left[m^{\prime}\right]=0$. This implies the first assertion. The second one follows directly from the divisibility of $\mathcal{N}^{0}(\mathcal{P})(k \llbracket t \rrbracket)$.

Finally, we consider the case where $\mathcal{P}^{\prime}=\widehat{\mathcal{P}}$ is the dual abelian variety of $\mathcal{P}$ and the isogeny $\phi$ is self-dual, that is, $\widehat{\phi}=\phi$. In this case, dualizing the short exact sequence (4.2.1) gives an isomorphism of $\Gamma$ with its Cartier dual, which in turn defines a non-degenerate pairing

$$
\langle\cdot, \cdot\rangle_{\phi}: \Gamma \times \Gamma \longrightarrow \mu_{n}
$$

compatible with the action of $\operatorname{Gal}(k((t)))=\widehat{\mu}$ on $\Gamma$. Here $n$ denotes the order of $\Gamma$. We will use the same notation for the pairing

$$
\langle\cdot, \cdot\rangle_{\phi}: \Gamma(k((t))) \times H^{1}(k((t)), \Gamma) \longrightarrow H^{1}\left(k((t)), \mu_{n}\right)=\operatorname{Hom}\left(\widehat{\mu}, \mu_{n}\right)=\mathbb{Z} / n \mathbb{Z}
$$

induced by taking cup-products. As in [Ogg62, Proposition 3], this pairing is again non-degenerate.

Lemma 4.2.2. With respect to the pairing (4.2.4), the groups $\Gamma^{0} \subset \Gamma(k((t)))$ and $\operatorname{Im}\left(\partial_{\phi}\right) \subset$ $H^{1}(k((t)), \Gamma)$ are exact annihilators of each other.

Proof. The Grothendieck pairing on component groups gives a perfect pairing between $\Phi_{\mathcal{P}}$ and $\Phi_{\widehat{\mathcal{P}}}$, see $\left[\mathrm{DRR}^{+} 72\right.$, Exposé IX]; in particular, $\left|\Phi_{\mathcal{P}}\right|=\left|\Phi_{\widehat{\mathcal{P}}}\right|$. From (4.2.2) and (4.2.3), we thus see that

$$
\left|\operatorname{Im}\left(\partial_{\phi}\right)\right|\left|\Gamma^{0}\right|=|\Gamma(k((t)))| .
$$

Hence, it is enough to show that $\langle\cdot, \cdot\rangle_{\phi}$ vanishes on $\Gamma^{0} \times \operatorname{Im}\left(\partial_{\phi}\right)$. For this, let $m=\left|\Phi_{\mathcal{P}}\right|=\left|\Phi_{\widehat{\mathcal{P}}}\right|$ and $\phi^{m}=\phi \circ[m]$ as in Lemma 4.2.1. Then $\phi^{m}$ is again self-dual, and, essentially by construction, we have the compatibility

$$
\left\langle x^{m}, y\right\rangle_{\phi}=\langle x, i(y)\rangle_{\phi^{m}},
$$

where $x \in \operatorname{ker}\left(\phi^{m}\right)(k((t))), y \in H^{1}(k((t)), \Gamma)$ and $i: H^{1}(k((t)), \Gamma) \rightarrow H^{1}\left(k((t)), \operatorname{ker}\left(\phi^{m}\right)\right)$. The lemma now follows directly from Lemma 4.2.1

Now, assume that $\underline{\Gamma}$ is constant over $k((t))$, which will be the only case of interest for us. In this case, we identify $\Gamma$ with its group of $k$-points. We further have an identification $H^{1}(k((t)), \Gamma) \cong \Gamma$ given by the choice of a primitive $|\Gamma|$ th root of unity; see Remark 3.1.3. The following corollary will be crucial for us.

Corollary 4.2.3. For any $\gamma \notin \operatorname{Im}\left(\partial_{\phi}\right)$, we have $\chi_{*, c}^{\varrho_{\gamma}}\left(\left[\mathcal{N}(\mathcal{P})_{k}\right]\right)=0$.

Proof. As $\mathcal{N}^{0}(\mathcal{P})_{k}$ is connected, the action of the finite group $\Gamma^{0}$ on the homological Chow motive $M\left(\mathcal{N}(\mathcal{P})_{k}\right)$ of $\mathcal{N}(\mathcal{P})_{k}$ is trivial by Corollary 2.5.3. On the other hand, Lemma 4.2.2 implies that $\varrho_{\gamma}$ is non-trivial on $\Gamma^{0}$ and thus $\chi_{*, c}^{\varrho_{\gamma}}\left(\left[\mathcal{N}(\mathcal{P})_{k}\right]\right)=0$. 


\section{Motivic integration On the Hitchin FiBRAtion}

\section{The Hausel-Thaddeus conjecture and a motivic version of topological mirror symmetry}

In this section, we recall some basic facts about Higgs bundles and state the topological mirror symmetry conjecture of Hausel-Thaddeus. For a more detailed account, we refer to their paper [HT03] and the references therein.

\subsection{Higgs bundles}

Let $C$ be a connected smooth projective curve of genus $g \geqslant 2$ over a field $k$ of characteristic zero. A Higgs bundle on $C$ is a pair $(E, \theta)$ consisting of a vector bundle $E \rightarrow C$ and a twisted endomorphism $\theta: E \rightarrow E \otimes K_{C}$, where $K_{C}$ denotes the canonical bundle of $C$.

Definition 5.1.1. Let $L \rightarrow C$ be a line bundle of degree $d \in \mathbb{Z}$.

(i) We denote by $\mathrm{M}_{n}^{L}$ the moduli space of semi-stable $L$-twisted $\mathrm{SL}_{n}$-Higgs bundles, that is, Higgs bundles $(E, \theta)$ of rank $n$ on $C$ together with an isomorphism $\operatorname{det} E \cong L$ satisfying $\operatorname{tr}(\theta)=0 \in H^{0}\left(C, K_{C}\right)$.

(ii) The finite group scheme $\Gamma=\operatorname{Pic}(C)[n] \equiv(\mathbb{Z} / n \mathbb{Z})^{2 g}$ of $n$-torsion line bundles on $C$ acts on $\mathrm{M}_{n}^{L}$ by tensoring the underlying vector bundle of a Higgs field. We write $\widehat{\mathbb{M}}_{n}^{d}=\left[\mathrm{M}_{n}^{L} / \Gamma\right]$ and $\widehat{\mathrm{M}}_{n}^{d}=\mathrm{M}_{n}^{L} / \Gamma$ for the stack and geometric quotient, respectively.

Remark 5.1.2. Note that $\widehat{\mathbb{M}}_{n}^{d}$ and $\widehat{\mathrm{M}}_{n}^{d}$ can be identified with a suitably defined moduli stack and space, respectively, of semi-stable $\mathrm{PGL}_{n}$-Higgs bundles of degree $d$; see $[\mathrm{GH} 13, \S 6]$. In particular, they depend only on $d$ and not on $L$.

We will only consider the case where $n$ and $d$ are coprime, in which case every semi-stable Higgs bundle is stable and $\mathrm{M}_{n}^{L}$ is a smooth quasi-projective variety.

\subsection{Hitchin fibrations and duality}

With a Higgs bundle $(E, \theta)$, one can associate its characteristic polynomial, whose $i$ th coefficient is given by $(-1)^{i} \operatorname{tr}\left(\wedge^{i} \theta\right) \in H^{0}\left(C, K_{C}^{i}\right)$. This defines morphisms

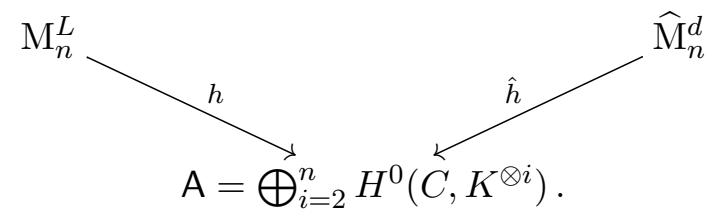

For $a \in \mathrm{A}$, we write $\mathrm{M}_{n, a}^{L}$ and $\widehat{\mathrm{M}}_{n, a}^{d}$ for the fibres $h^{-1}(a)$ and $\hat{h}^{-1}(a)$, respectively. To describe the generic fibres of $h$ and $\hat{h}$, we use the spectral curve construction. For any $a=\left(a_{i}\right)_{2 \leqslant i \leqslant n} \in \mathrm{A}$, the spectral curve $C_{a}$ is the subscheme inside the total space of $K_{C}$ defined by the equation

$$
\left\{X^{n}+a_{2} X^{n-2}+\cdots+a_{n}=0\right\} \subset \operatorname{Tot}\left(K_{C}\right) .
$$

There is a non-empty open subvariety $\mathrm{A}^{\mathrm{sm}} \subset \mathrm{A}$ defined by the condition that $C_{a}$ is smooth and geometrically connected. For $a \in \mathrm{A}^{\mathrm{sm}}$, the projection $\pi: C_{a} \rightarrow C$ is a ramified cover of degree $n$ and hence induces a degree-preserving norm map

$$
\mathrm{Nm}: \operatorname{Pic}\left(C_{a}\right) \longrightarrow \operatorname{Pic}(C) \text {. }
$$

The Prym variety $\mathrm{P}_{a}$ is defined as the kernel of Nm. It is an abelian variety, and its dual $\widehat{\mathrm{P}}_{a}$ can be identified with the quotient $\mathrm{P}_{a} / \Gamma$, where the inclusion $\pi^{*}: \Gamma \rightarrow \mathrm{P}_{a}$ is defined by pullback 


\section{F. LOESER AND D. WYSS}

along $\pi: C_{a} \rightarrow C$. We quickly explain this duality; see also [HT03, Lemma 2.3]. By definition, there is a short exact sequence

$$
0 \longrightarrow \mathrm{P}_{a} \longrightarrow \operatorname{Pic}^{0}\left(C_{a}\right) \stackrel{\mathrm{Nm}}{\longrightarrow} \mathrm{Pic}^{0}(C) \longrightarrow 0
$$

Dualizing the sequence and using the auto-duality of $\mathrm{Pic}^{0}$, we get

$$
0 \longrightarrow \operatorname{Pic}^{0}(C) \stackrel{\pi^{*}}{\longrightarrow} \operatorname{Pic}^{0}\left(C_{a}\right) \longrightarrow \widehat{\mathrm{P}}_{a} \longrightarrow 0 \text {. }
$$

Finally, there is an isomorphism $\operatorname{Pic}^{0}\left(C_{a}\right) / \operatorname{Pic}^{0}(C) \cong \mathrm{P}_{a} / \Gamma$ induced by the morphism

$$
\mathrm{Pic}^{0}\left(C_{a}\right) \longrightarrow \mathrm{P}_{a} / \Gamma, \quad M \longmapsto M \otimes \pi^{*} \mathrm{Nm}\left(M^{-1}\right)^{1 / n},
$$

where $(\cdot)^{1 / n}: \mathrm{Pic}^{0}(C) \stackrel{\sim}{\rightarrow} \operatorname{Pic}^{0}(C) / \Gamma$ denotes the isomorphism induced by the $n$th power map. In particular, the quotient $\mathrm{P}_{a} \rightarrow \mathrm{P}_{a} / \Gamma$ factors as

$$
\mathrm{P}_{a} \longrightarrow \mathrm{Pic}^{0}\left(C_{a}\right) \longrightarrow \mathrm{P}_{a} / \Gamma
$$

and is therefore self-dual.

Proposition 5.2.1 ([BNR89, Proposition 3.6]). For any $a \in \mathrm{A}^{\mathrm{sm}}$, we have isomorphisms

$$
\mathrm{M}_{n, a}^{L} \cong(\mathrm{Nm})^{-1}(L) \text { and } \widehat{\mathrm{M}}_{n, a}^{d} \cong(\mathrm{Nm})^{-1}(L) / \Gamma \cong \operatorname{Pic}^{d}\left(C_{a}\right) / \operatorname{Pic}^{0}(C) .
$$

In particular, $\mathrm{M}_{n, a}^{L}$ and $\widehat{\mathrm{M}}_{n, a}^{d}$ are torsors for $\mathrm{P}_{a}$ and $\widehat{\mathrm{P}}_{a}$, respectively.

In [HT03, Theorem 3.7], Hausel and Thaddeus extend the duality between $\mathrm{P}_{a}$ and $\widehat{\mathrm{P}}_{a}$ to a duality between the torsors $\mathrm{M}_{n, a}^{L}$ and $\widehat{\mathrm{M}}_{n, a}^{d}$ and interpret this as (twisted) SYZ-mirror symmetry of $\mathrm{M}_{n}^{L}$ and $\widehat{\mathrm{M}}_{n}^{d^{\prime}}$. This was their motivation for conjecturing Theorem 5.3.1 below.

Remark 5.2.2 (Volume forms). One can extend the construction of the Prym variety to all of $\mathrm{A}$ and obtain a group scheme $\mathrm{P} \rightarrow \mathrm{A}$ acting on $\mathrm{M}_{n}^{L}$. The restriction $\mathrm{M}_{n}^{L \text {,red }}$ of $\mathrm{M}_{n}^{L}$ to $\mathrm{A}^{\text {red }}=\{a \in$ A $\mid C_{a}$ is reduced $\}$ contains an open $\widetilde{\mathrm{M}}_{n}^{L} \subset \mathrm{M}_{n}^{L, \text { red }}$ which is a torsor under Pred and is dense in every Hitchin fibre over $A^{\text {red; }}$ see [Ngô10, Proposition 4.16.1]. As codim $A \backslash A^{\text {red }} \geqslant 2$, the same is true for $\mathrm{M}_{n}^{L} \backslash \widetilde{\mathrm{M}}_{n}^{L}$, and we use this to construct an explicit $\Gamma$-invariant volume form $\omega^{L}$ on $\mathrm{M}_{n}^{L}$, as follows.

Let $\omega_{\mathrm{A}}$ be a volume form on the affine space $A$ and $\omega_{P}$ a translation-invariant trivializing section of the relative canonical bundle $K_{\mathrm{P} / \mathrm{A}}$. Then $\omega_{\mathrm{P}} \wedge \omega_{\mathrm{A}}$ is a global volume form on $\mathrm{P}$ which induces one on the torsor $\widetilde{\mathrm{M}}_{n}^{L}$; see [GWZ20, Lemma 6.13]. As we have codim $\mathrm{M}_{n}^{L} \backslash \widetilde{\mathrm{M}}_{n}^{L} \geqslant 2$, this form extends to a volume form $\omega^{L}$ on $\mathrm{M}_{n}^{L}$.

\subsection{The main result}

Let $\langle\cdot, \cdot\rangle: \Gamma \times \Gamma \rightarrow \mu_{n}$ be the Weil pairing on $\Gamma=\operatorname{Pic}^{0}(C)[n]$. We write $\varrho \in H_{\text {grp }}^{2}\left(\Gamma, \mu_{n}\right)$ for the class defined by $\langle\cdot, \cdot\rangle$ viewed as a 2-cocycle, and for $\gamma \in \Gamma$, we write $\varrho_{\gamma}=\langle\gamma, \cdot\rangle: \Gamma \rightarrow \mu_{n}$ for the character induced by $\gamma$.

The following theorem was conjectured by Hausel and Thaddeus in [HT03, Conjecture 5.1] and proven by Groechenig, Ziegler and the second author.

Theorem 5.3.1 ([GWZ20, Theorem 7.21]). Assume $k=\mathbb{C}$. Let $d$, $d^{\prime}$ be integers prime to $n$ and $L, L^{\prime}$ line bundles on $C$ of degree $d$ and $d^{\prime}$, respectively. Let $q$ be the multiplicative inverse of $d^{\prime}$ modulo $n$. Then we have

$$
E\left(\mathrm{M}_{n}^{L} ; x, y\right)=E_{\mathrm{st}}^{\varrho^{-d q}}\left(\widehat{\mathrm{M}}_{n}^{d^{\prime}} ; x, y\right)
$$




\section{Motivic integration On the Hitchin FiBRAtion}

Remarks 5.3.2. (i) In the original conjecture, the $E$-polynomial on the right-hand side is twisted by a $\mu_{n}$-gerbe on $\widehat{\mathbb{M}}_{n}^{d^{\prime}}$, whereas we twist by the pullback of a $\mu_{n}$-gerbe on $[\operatorname{Spec}(k) / \Gamma]$. The equivalence of the two formulations is mentioned at the end of Section 4 in [HT03] and proven in the case when $n$ is prime in Proposition 8.1 of that paper. In the appendix, we explain how to deduce this equivalence in general.

(ii) Our proof will show that both sides of (5.3.1) are independent of $L, L^{\prime}$ and their degrees.

(iii) There is a natural $\mathbb{C}^{*}$-action on $\mathrm{M}_{n}^{L}$ given by scaling the Higgs field, which commutes with the $\Gamma$-action. This gives $\mathrm{M}_{n}^{L}$ and $\mathrm{M}_{n}^{L, \gamma}$, for $\gamma \in \Gamma$, the structure of semi-projective varieties; see [HR15]. In particular, their cohomologies are pure [HR15, Corollary 1.3.2]. Hence, the equality (1.1.1) stated in the introduction follows from (5.3.1).

We now formulate the main theorem of this article, an equality between virtual motives, which will imply Theorem 5.3.1.

THEOREM 5.3.3. Let $k$ be an algebraically closed field of characteristic zero. Let $d, d^{\prime}$ and $q$ be integers prime to $n$, and let $L$ and $L^{\prime}$ be line bundles on $C$ of degree $d$ and $d^{\prime}$, respectively. Then we have

$$
\mathbb{L}^{-\operatorname{dim} \mathrm{M}_{n}^{L}} \vartheta_{*_{k}}\left(\left[\mathrm{M}_{n}^{L}\right]\right)=\sum_{\gamma \in \Gamma} \mathbb{L}^{-w(\gamma)} \vartheta_{*_{k}}^{\varrho_{\gamma}^{-q}}\left(\left[\mathrm{M}_{n}^{L^{\prime}, \gamma}\right]\right)
$$

in $\mathcal{C}_{\text {mot }}\left(*_{k}\right)$.

In particular, when $L=L^{\prime}$ and $q=1$, one recovers Theorem 1.3.1 from the introduction. Theorem 5.3.1 follows from Theorem 5.3.3 by taking the E-polynomial of both sides of (5.3.2). Indeed, $E\left(\vartheta_{*_{k}}\left(\left[\mathrm{M}_{n}^{L}\right]\right)\right)=E\left(\mathrm{M}_{n}^{L} ; x, y\right)$ and $E\left(\vartheta_{*_{k}}^{\varrho_{\gamma}^{-q}}\left(\left[\mathrm{M}_{n}^{L^{\prime}, \gamma}\right]\right)\right)=E^{\varrho_{\gamma}^{-q}}\left(\mathrm{M}_{n}^{L^{\prime}, \gamma} ; x, y\right)$, by the compatibility between (equivariant) $E$-polynomials of varieties and $E$-polynomials of their Chow motives recalled in $\S 3.3 .3$.

Remark 5.3.4. The left-hand side of (5.3.2) admits a decomposition into $\Gamma$-isotypical components, and our argument in Section 6 in fact shows that for any $\gamma \in \Gamma$,

$$
\mathbb{L}^{-\operatorname{dim} \mathrm{M}_{n}^{L}} \vartheta_{*_{k}}^{\varrho_{\gamma}^{-q}}\left(\left[\mathrm{M}_{n}^{L}\right]\right)=\mathbb{L}^{-w(\gamma)} \vartheta_{*_{k}}^{\varrho_{\gamma}^{-q}}\left(\left[\mathrm{M}_{n}^{L^{\prime}, \gamma}\right]\right) .
$$

On the level of $E$-polynomials, this refined version of topological mirror symmetry has been conjectured by Hausel (unpublished) and can be deduced by a Fourier-transform argument in the $p$-adic setting [GWZ20, Theorem 7.24].

\subsection{Proof of Theorem 5.3.3}

We will deduce Theorem 5.3.3 from an equality between motivic integrals that we will prove in the next section.

Fix an algebraically closed field $k$ of characteristic zero. We will work on the constant pullbacks $\mathcal{M}_{n}^{L}=\mathrm{M}_{n}^{L} \times_{k} \operatorname{Spec}(k \llbracket t \rrbracket)$ and $\widehat{\mathcal{M}}_{n}^{d}=\widehat{\mathrm{M}}_{n}^{d} \times_{k} \operatorname{Spec}(k \llbracket t \rrbracket)=\mathcal{M}_{n}^{L} / \Gamma$, which by functoriality parametrize twisted Higgs bundles on $\mathcal{C}=C \times_{k} \operatorname{Spec}(k \llbracket t \rrbracket)$. Let

$$
e_{d}: \widehat{\mathcal{M}}_{n}^{d, \natural} \rightarrow \underline{I \mathrm{M}_{n}^{L}}=\bigsqcup_{\gamma \in \Gamma} \underline{\mathrm{M}_{n}^{L, \gamma} / \Gamma}
$$

be the specialization morphism from Construction 3.1.2 and $\bar{e}_{d}: \widehat{\mathcal{M}}_{n}^{d, \natural} \rightarrow \Gamma$ the composition of $e_{d}$ with the map to the index set of the disjoint union. 


\section{F. LOESER AND D. WYSS}

Finally, let $\omega^{L}$ be the $\Gamma$-invariant global volume form on $\mathcal{M}_{n}^{L}$ from Remark 5.2.2 and $\omega_{\text {orb }}^{d}$ its quotient on $\widehat{\mathcal{M}}_{n}^{d}$.

Theorem 5.4.1. Let $d, d^{\prime}$ and $q$ be integers prime to $n$ and $L, L^{\prime}$ line bundles on $C$ of degree $d$ and $d^{\prime}$, respectively. Then we have

$$
\int_{{\underline{\mathcal{M}_{n}}}_{n_{0}}^{L}}^{\text {mot }}\left|\omega^{L}\right|=\sum_{\gamma \in \Gamma} \int_{\bar{e}_{d^{\prime}}^{-1}(\gamma)}^{\text {mot, } \varrho_{\gamma}^{-q}} \mathcal{M}_{n}^{L^{\prime}, \natural} \times^{\Gamma} T_{\gamma^{-1}}\left|\omega_{\text {orb }}^{d^{\prime}}\right|
$$

in $\mathcal{C}_{\text {mot }}\left(*_{k}\right)$.

Theorem 5.4.1 directly implies Theorem 5.3.3 by applying respectively Lemma 3.1.1 and Theorem 3.1.6 to the left-hand side and to the right-hand side of (5.4.1).

\section{Proof of Theorem 5.4.1}

We will write $\mathcal{A}, \mathcal{A}^{\mathrm{sm}}, \mathcal{P}, \widehat{\mathcal{P}}$, etc. for the constant pullbacks of the constructions of Section 5.2 from $k$ to $k \llbracket t \rrbracket$.

\subsection{Reduction by a Fubini theorem}

We first reduce (5.4.1) to a comparison of Hitchin fibres. For this, consider the assignment $\mathcal{A}^{\mathrm{b}}=\underline{\mathcal{A}} \cap \underline{\mathcal{A}_{k((t))}^{\mathrm{sm}}}$, that is, for $\bar{K} / k$ algebraically closed,

$$
\mathcal{A}^{b}(\bar{K})=\mathcal{A}(\bar{K} \llbracket t \rrbracket) \cap \mathcal{A}^{\mathrm{sm}}(\bar{K}((t))) .
$$

Since the complement of $h^{-1}\left(\mathcal{A}^{b}\right)$ in $\mathcal{M}_{n_{\circ}}^{L}$ has smaller $K$-dimension than $\mathcal{M}_{n_{\circ}}^{L}$, it has measure 0 , as follows from facts recalled in the first paragraph of $\S 2.2 .4$, and thus by Proposition 2.3.2, we have

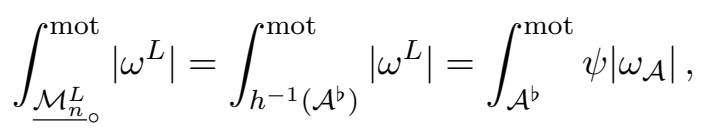

where, for any point $a \in\left|\mathcal{A}^{b}\right|$,

$$
\psi(a)=\int_{\underline{\mathcal{M}_{n, a}^{L}}}^{\text {mot }}\left|\omega_{a}^{L}\right| .
$$

Here by construction, see Remark 5.2.2, the form $\omega_{a}^{L}$ is a translation-invariant global form on the $\mathcal{P}_{a}$-torsor $\mathcal{M}_{n, a}^{L}$. We use the properness of $h$ to identify $\underline{\mathcal{M}_{n, a}^{L}}$ and $\underline{\mathcal{M}_{n, a}^{L}}$.

Similarly, the complement of $\hat{h}^{-1}\left(\mathcal{A}^{b}\right)$ in $\widehat{\mathcal{M}}_{n}^{d, \natural}$ has measure 0 , and we can rewrite the righthand side of (5.4.1) using Proposition 2.4.3:

$$
\sum_{\gamma \in \Gamma} \int_{\bar{e}_{d^{\prime}}^{-1}(\gamma)}^{\text {mot, } \varrho_{\gamma}^{-q}} \mathcal{M}_{n}^{L^{\prime}, \natural} \times{ }^{\Gamma} T_{\gamma^{-1}}\left|\omega_{\text {orb }}^{d^{\prime}}\right|=\int_{\mathcal{A}^{b}}^{\text {mot }} \psi^{\prime}\left|\omega_{\mathcal{A}}\right| .
$$

Here for any $a \in\left|\mathcal{A}^{b}\right|$, the evaluation of the morphism $\psi^{\prime}$ at $a$ is given by

$$
\psi^{\prime}(a)=\sum_{\gamma \in \Gamma} \int_{\bar{e}_{d^{\prime}, a}^{-1}(\gamma)}^{\text {mot, } \varrho_{\gamma}^{-q}} \underline{\mathcal{M}_{n, a}^{L^{\prime}} \times \Gamma T_{\gamma^{-1}}}\left|\omega_{\text {orb }, a}^{d^{\prime}}\right|,
$$

where we put

$$
\bar{e}_{d^{\prime}, a}=\bar{e}_{d^{\prime} \mid \widehat{\mathcal{M}}_{n, a}^{d}}: \widehat{\mathcal{M}}_{n, a}^{d} \longrightarrow \Gamma
$$




\section{Motivic Integration ON THE Hitchin FibRATion}

Combining the two sides of (5.4.1), we see that Theorem 5.4.1 is now a consequence of the following theorem.

Theorem 6.1.1. For every $a \in\left|\mathcal{A}^{b}\right|$, we have

$$
\int_{\underline{\mathcal{M}_{n, a}^{L}}}^{\text {mot }}\left|\omega_{a}^{L}\right|=\sum_{\gamma \in \Gamma} \int_{\bar{e}_{d^{\prime}, a}^{-1}(\gamma)}^{\text {mot, }, e_{\gamma}^{-q}} \underline{\mathcal{M}_{n, a}^{L^{\prime}} \times{ }^{\Gamma} T_{\gamma^{-1}}}\left|\omega_{\text {orb }, a}^{d^{\prime}}\right|
$$

in $\mathcal{C}_{\text {mot }}\left(*_{k(a)}\right)$.

\subsection{Independence of $\boldsymbol{d}$}

Proposition 6.2.1. Let $L$ and $L^{\prime}$ be line bundles on $C$ of degree $d$ and $d^{\prime}$, both prime to $n$. Then for every $a \in\left|\mathcal{A}^{b}\right|$, we have

$$
\int_{\underline{\mathcal{M}_{n, a}^{L}}}\left|\omega_{a}^{L}\right|=\int_{\underline{\mathcal{M}_{n, a}^{L^{\prime}}}}\left|\omega_{a}^{L^{\prime}}\right|
$$

in $\mathcal{C}_{\text {acf }}\left(*_{k(a)}\right)$.

Proof. Recall that a point $a \in\left|\mathcal{A}^{b}\right|$ corresponds to a morphism $\operatorname{Spec}(K \llbracket t \rrbracket) \rightarrow \mathcal{A}$ whose generic fibre lies in $\mathcal{A}^{\mathrm{sm}}$, where $K$ is a field extension of $k$. In particular, by Proposition 5.2.1, both $\mathcal{M}_{n, a}^{L}$ and $\mathcal{M}_{n, a}^{L^{\prime}}$ are $\mathcal{P}_{a}$-torsors over $K((t))$, and if there is a trivialization $\tau: \mathcal{P}_{a} \rightarrow \mathcal{M}_{n, a}^{L}$, then $\tau^{*} \omega_{a}^{L}=\omega_{\mathcal{P}, a}$ by construction; see Remark 5.2.2. Thus the proposition follows if we can show that $\mathcal{M}_{n, a}^{L}$ has a $K((t))$-rational point if and only if $\mathcal{M}_{n, a}^{L^{\prime}}$ has one.

To see this, we use the identifications $\mathcal{M}_{n, a}^{L} \cong \mathrm{Nm}^{-1}(L)$ and $\mathcal{M}_{n, a}^{L^{\prime}} \cong \mathrm{Nm}^{-1}\left(L^{\prime}\right)$ from Proposition 5.2.1, where by abuse of notation we also write $L, L^{\prime}$ for the line bundles on $C \times{ }_{k} \operatorname{Spec}(K \llbracket t \rrbracket)$. We take an integer $e$ such that $d e \equiv d^{\prime} \bmod n$. Then by [GWZ20, Lemma 5.8], we have

$$
\left[\mathrm{Nm}^{-1}\left(L^{\prime}\right)\right]\left[\mathrm{Nm}^{-1}\left(L^{-1}\right)\right]^{e}=\left[\mathrm{Nm}^{-1}\left(L^{\prime} \otimes L^{-e}\right)\right] \in H^{1}\left(K((t)), \mathcal{P}_{a}\right) .
$$

Since the degree of the line bundle $L^{\prime} \otimes L^{-e} \in \operatorname{Pic}(C)$ is divisible by $n$, it admits an $n$th root (the base field $k$ is algebraically closed), from which we deduce $\left[\mathrm{Nm}^{-1}\left(L^{\prime} \otimes L^{-e}\right)\right]=0$; see again [GWZ20, Lemma 5.8]. This shows that $\mathcal{M}_{n, a}^{L^{\prime}}$ admits a $K((t))$-rational point if $\mathcal{M}_{n, a}^{L}$ does. Interchanging the roles of $d$ and $d^{\prime}$ proves the converse and the proposition.

Corollary 6.2.2. Let $L$ and $L^{\prime}$ be line bundles on $C$ of degree $d$ and $d^{\prime}$ prime to $n$. Then we have $\left[\mathrm{M}_{n}^{L}\right]=\left[\mathrm{M}_{n}^{L^{\prime}}\right] \in \mathcal{M}_{k} \otimes_{\mathbb{Z}[\mathbb{L}]} \mathbb{A}$.

\subsection{End of the proof}

Because of Proposition 6.2.1, it is enough to prove Theorem 6.1.1 under the assumption $L=L^{\prime}$. Hence, we have a quotient morphism $\mathcal{M}_{n}^{L} \rightarrow \widehat{\mathcal{M}}_{n}^{d}$, and we may assume that $a \in \mathcal{A}^{b}$ is such that $\widehat{\mathcal{M}}_{n, a}^{d}$ is an unramified $\widehat{\mathcal{P}}_{a}$-torsor. Indeed, otherwise both $\mathcal{M}_{n, a}^{L}$ and $\widehat{\mathcal{M}}_{n, a}^{d}$ are the empty assignment, and both sides of (6.1.1) are 0. We will write $K$ for the residue field $k(a)$.

We start by computing the left-hand side of (6.1.1).

Proposition 6.3.1. If $\mathcal{M}_{n, a}^{L}$ is an unramified $\mathcal{P}_{a}$-torsor, we have

$$
\int_{\mathcal{M}_{n, a}^{L}}^{\text {mot }}\left|\omega_{a}^{L}\right|=\mathbb{L}^{-\operatorname{ord} \mathcal{N}_{a}^{L}}\left[\mathcal{N}\left(\mathcal{M}_{n, a}^{L}\right)_{K}\right] \in \mathcal{C}_{\operatorname{mot}}\left(*_{K}\right),
$$




\section{F. LOESER AND D. WYSS}

where ord $\mathcal{N}_{\omega_{a}^{L}} \in \mathbb{Z}$ is the order of vanishing of $\omega_{a}^{L}$ along the special fibre of $\mathcal{N}\left(\mathcal{M}_{n, a}^{L}\right)_{K}$; see Remark 4.1.1. If $\mathcal{M}_{n, a}^{L}$ is not unramified, then

$$
\int_{\underline{\mathcal{M}_{n, a}^{L}}}^{\operatorname{mot}}\left|\omega_{a}^{L}\right|=0
$$

Proof. If $\mathcal{M}_{n, a}^{L}$ is unramified, then $\mathcal{N}\left(\mathcal{M}_{n, a}^{L}\right)$ is a smooth model of $\mathcal{M}_{n, a}^{L}$ over $\operatorname{Spec}(K \llbracket t \rrbracket)$. Furthermore, by the Néron mapping property, the subassignments associated with $\mathcal{M}_{n, a}^{L}$ and $\mathcal{N}\left(\mathcal{M}_{n, a}^{L}\right)$ are isomorphic; hence, we can argue as in Remark 4.1.1 to conclude.

If $\mathcal{M}_{n, a}^{L}$ is not unramified, $\underline{\mathcal{M}}_{n, a}^{L}$ is the empty assignment and thus $\int_{\underline{\mathcal{M}}_{n, a}^{L}}^{\text {mot }}\left|\omega_{a}^{L}\right|=0$.

We are left with computing the right-hand side of (6.1.1) in the two cases appearing in Proposition 6.3.1. For this, let $\partial$ be the connecting homomorphism in the long exact sequence

$$
0 \longrightarrow \Gamma \longrightarrow \mathcal{P}_{a}(K((t))) \longrightarrow \widehat{\mathcal{P}}_{a}(K((t))) \stackrel{\partial}{\longrightarrow} H^{1}(K((t)), \Gamma) \longrightarrow \cdots .
$$

Recall from Remark 3.1.3 that we have $H^{1}(K((t)), \Gamma) \cong H^{1}(K, \Gamma) \oplus \Gamma$. With respect to this decomposition, we write $\partial=\partial^{u r} \oplus \bar{\partial}$. As in Proposition 3.1.4, one sees that $\bar{\partial}$ extends to a definable morphism

$$
\bar{\partial}: \widehat{\mathcal{P}}_{a} \longrightarrow \Gamma .
$$

Notice that $\bar{\partial}$ and $\bar{e}_{d, a}$ are closely related, as the following lemma shows.

Lemma 6.3.2. The $\mathcal{P}_{a}$-torsor $\mathcal{M}_{n, a}^{L}$ is unramified if and only if the images of $\bar{\partial}$ and $\bar{e}_{d, a}$, when evaluated over $\bar{K}$, are equal as subsets of $\Gamma$, that is,

$$
\operatorname{Im}\left(\bar{e}_{d, a}\right)(\bar{K})=\operatorname{Im}(\bar{\partial})(\bar{K}) .
$$

Proof. Notice that $\operatorname{Im}\left(\bar{e}_{d, a}\right)(\bar{K})$ is a coset for $\operatorname{Im}(\bar{\partial})(\bar{K})$ since the actions of $\mathcal{P}_{a}$ and $\widehat{\mathcal{P}}_{a}$ on the respective Hitchin fibres are compatible with the quotient map $\mathcal{M}_{n}^{L} \rightarrow \widehat{\mathcal{M}}_{n}^{d}$. The lemma now follows from the observation that $\mathcal{M}_{n, a}^{L}$ is unramified if and only if $\operatorname{Im}\left(\bar{e}_{d, a}\right)(\bar{K})$ contains the trivial torsor.

Proposition 6.3.3 (Unramified case). Assume that $\mathcal{M}_{n, a}^{L}$ is unramified. Then we have

$$
\sum_{\gamma \in \Gamma} \int_{\bar{e}_{d, a}^{-1}(\gamma)}^{\text {mot, } \varrho_{\gamma}^{-q}} \underline{\mathcal{M}_{n, a}^{L} \times{ }^{\Gamma} T_{\gamma^{-1}}}\left|\omega_{\text {orb }, a}^{d}\right|=\mathbb{L}^{-\operatorname{ord} \mathcal{N}_{a}^{L}}\left[\mathcal{N}\left(\mathcal{M}_{n, a}^{L}\right)_{K}\right] \in \mathcal{C}_{\operatorname{mot}}\left(*_{K}\right) .
$$

Proof. We fix $\gamma \in \operatorname{Im}\left(\bar{e}_{d, a}\right)(\bar{K})=\operatorname{Im}(\bar{\partial})(\bar{K}) \subset \Gamma$ (otherwise, the corresponding summand on the left-hand side is 0$)$. By construction, $\bar{e}_{d, a}^{-1}(\gamma) \subset \underline{\mathcal{M}}_{n, a}^{d}$ is exactly the support of the function induced by $\mathcal{M}_{n, a}^{L} \times^{\Gamma} T_{\gamma^{-1}}$. Thus we have

$$
\int_{\bar{e}_{d, a}^{-1}(\gamma)}^{\text {mot, } \varrho_{\gamma}^{-q}} \underline{\mathcal{M}_{n, a}^{L} \times{ }^{\Gamma} T_{\gamma^{-1}}}\left|\omega_{\text {orb }, a}^{d}\right|=\int_{\widehat{\mathcal{M}}_{n, a}^{d}}^{\text {mot, } \varrho_{\gamma}^{-q}} \underline{\mathcal{M}_{n, a}^{L} \times{ }^{\Gamma} T_{\gamma^{-1}}}\left|\omega_{\text {orb }, a}^{d}\right| .
$$

But since

$$
\varrho_{\gamma}^{-q}\left(\gamma^{-1}\right)=\langle\gamma, \gamma\rangle^{q}=1
$$

the $\mu_{n}$-torsor $\left(\varrho_{\gamma}^{-q}\right)_{*} T_{\gamma^{-1}}$ is trivial, and therefore the functions induced by $\mathcal{M}_{n, a}^{L}$ and $\mathcal{M}_{n, a}^{L} \times{ }^{\Gamma} T_{\gamma^{-1}}$ have the same $\varrho_{\gamma}^{-q}$-isotypical component by Lemma 2.7.2. As $\mathcal{M}_{n, a}^{L}$ is unramified by assumption, 


\section{Motivic Integration ON THE Hitchin FibRATion}

from Lemma 2.6.2, we get

$$
\int_{\underline{\widehat{\mathcal{M}}_{n, a}^{d}}}^{\operatorname{mot}, \varrho_{\gamma}^{-q}} \underline{\mathcal{M}_{n, a}^{L} \times{ }^{\Gamma} T_{\gamma^{-1}}}\left|\omega_{\text {orb }, a}^{d}\right|=\int_{{\widehat{\mathcal{M}_{n, a}^{d}}}^{\operatorname{mot}, \varrho_{\gamma}^{-q}}}^{\mathcal{M}_{n, a}^{L}}\left|\omega_{\text {orb }, a}^{d}\right|=\chi_{*, c}^{\varrho_{\gamma}^{-q}} \mathbb{L}^{-\operatorname{ord} \mathcal{N} \omega_{a}^{L}}\left[\mathcal{N}\left(\mathcal{M}_{n, a}^{L}\right)_{K}\right] .
$$

On the other hand, we have a decomposition

$$
\left[\mathcal{N}\left(\mathcal{M}_{n, a}^{L}\right)_{K}\right]=\sum_{\gamma \in \Gamma} \chi_{*, c}^{\varrho_{\gamma}}\left[\mathcal{N}\left(\mathcal{M}_{n, a}^{L}\right)_{K}\right]=\sum_{\gamma \in \operatorname{Im}(\bar{\partial})(\bar{K})} \chi_{*, c}^{\varrho_{\gamma}}\left[\mathcal{N}\left(\mathcal{M}_{n, a}^{L}\right)_{K}\right]
$$

where the last equality follows from Corollary 4.2.3. As the order of $\gamma$ divides $n$ and $q$ is prime to $n$, the map $\gamma \mapsto \gamma^{-q}$ induces a bijection on $\operatorname{Im}(\bar{\partial})$, and the proposition follows.

Proposition 6.3.4 (Ramified case). If $\mathcal{M}_{n, a}^{L}$ is not unramified, we have

$$
\sum_{\gamma \in \Gamma} \int_{\bar{e}_{d, a}^{-1}(\gamma)}^{\text {mot, } \varrho_{\gamma}^{-q}} \underline{\mathcal{M}_{n, a}^{L} \times \Gamma T_{\gamma^{-1}}}\left|\omega_{\text {orb }, a}^{d}\right|=0
$$

Proof. We show that each summand on the left-hand side is 0 . If $\gamma \notin \operatorname{Im}\left(\bar{e}_{d, a}\right)(\bar{K})$, this is clear, as then $\bar{e}_{d, a}^{-1}(\gamma)$ is empty, so we fix $\gamma \in \operatorname{Im}\left(\bar{e}_{d, a}\right)(\bar{K})$.

First we claim that $\mathcal{M}_{n, a}^{L} \times{ }^{\Gamma} T_{\gamma^{-1}}$ is unramified. To see this, we pick a point $x \in \widehat{\mathcal{M}}_{n, a}^{d}(\bar{K}((t)))$ with $\bar{e}_{d, a}(x)=\gamma$. The fibre $T_{x}$ of the projection $\mathcal{M}_{n, a}^{L} \rightarrow \widehat{\mathcal{M}}_{n, a}^{d}$ is a $\Gamma$-torsor isomorphic to $T_{\gamma}$, and $\mathcal{M}_{n, a}^{L} \cong \mathcal{P}_{a} \times{ }^{\Gamma} T_{x}$ since $\mathcal{M}_{n, a}^{L}$ is a $\mathcal{P}_{a}$-torsor. Hence, over $\bar{K}((t))$ we find

$$
\mathcal{M}_{n, a}^{L} \times{ }^{\Gamma} T_{\gamma^{-1}} \cong\left(\mathcal{P}_{a} \times{ }^{\Gamma} T_{x}\right) \times{ }^{\Gamma} T_{\gamma^{-1}} \cong \mathcal{P}_{a}
$$

which shows that $\mathcal{M}_{n, a}^{L} \times^{\Gamma} T_{\gamma^{-1}}$ is unramified. By Lemma 2.6.2, we then get

$$
\int_{\bar{e}_{d, a}^{-1}(\gamma)}^{\text {mot, } \varrho_{\gamma}^{-q}} \underline{\mathcal{M}_{n, a}^{L} \times{ }^{\Gamma} T_{\gamma^{-1}}}\left|\omega_{\text {orb }, a}^{d}\right|=\chi_{*, c}^{\varrho_{\gamma}^{-q}} \mathbb{L}^{-\operatorname{ord} \mathcal{N}_{\mathcal{P}, a}}\left[\mathcal{N}\left(\mathcal{M}_{n, a}^{L} \times^{\Gamma} T_{\gamma^{-1}}\right)_{K}\right] .
$$

By Lemma 6.3.2, we know that $\gamma$ and thus also $\gamma^{-q}$ are not in $\operatorname{Im}(\bar{\partial})(\bar{K})$, and thus Corollary 4.2.3 implies

$$
\chi_{*, c}^{\varrho_{\gamma}^{-q}}\left[\mathcal{N}\left(\mathcal{M}_{n, a}^{L} \times^{\Gamma} T_{\gamma^{-1}}\right)_{K}\right]=0,
$$

which is what we needed to show.

Proof of Theorem 6.1.1. We just need to summarize the previous calculations. First if $a \in \mathcal{A}^{b}$ is such that $\mathcal{M}_{n, a}^{L}$ is not unramified, we deduce from Propositions 6.3.1 and 6.3.4 that both sides of (6.1.1) are 0. If $\mathcal{M}_{n, a}^{L}$ is unramified, we similarly obtain that both sides of (6.1.1) equal

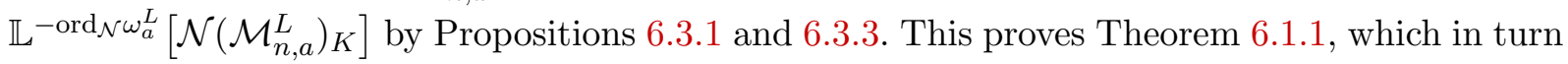
implies Theorem 5.4.1.

\section{Appendix. Comparison of gerbes}

For the whole appendix, we assume $k=\mathbb{C}$ and use the notation of Section 5. In particular, $L$ denotes a line bundle of degree $d$ on $C$, and $n$ is a positive integer prime to $d$. We further fix a point $c \in C$.

We start by recalling the construction in [HT03, §3] of $\mu_{n}$-gerbes $\alpha_{L}$ and $\widehat{\alpha}_{L}$ on $\mathrm{M}_{n}^{L}$ and $\widehat{\mathbb{M}}_{n}^{d}$, respectively. Since we assume $(n, d)=1$, there exists a universal $L$-twisted Higgs bundle

$$
(\mathbf{E}, \boldsymbol{\theta}) \longrightarrow \mathrm{M}_{n}^{L} \times C
$$




\section{F. LOESER AND D. WYSS}

and we denote the restriction to $\mathrm{M}_{n}^{L} \times\{c\}$ by the same letters. The gerbe $\alpha_{L}$ is then defined as the gerbe of liftings of the projectivization $\mathbb{P E}$ of $\mathbf{E}$ to an honest $\mathrm{SL}_{n}$-bundle; that is, for any étale neighbourhood $p: U \rightarrow \mathrm{M}_{n}^{L}$, the category $\alpha_{L}(U)$ consists of pairs $(F, \phi)$ with $F \rightarrow U$ a vector bundle of rank $n$ and trivial determinant and $\phi: \mathbb{P} F \stackrel{\sim}{\rightarrow} p^{*} \mathbb{P} \mathbf{E}$ an isomorphism. Tensoring by $\mu_{n}$-torsors gives $\alpha_{L}$ the structure of a $\mu_{n}$-gerbe.

Now $\widehat{\alpha}_{L}$ on the quotient stack $\widehat{\mathbb{M}}_{n}^{d}=\left[\mathrm{M}_{n}^{L} / \Gamma\right]$ is simply $\alpha_{L}$ together with a $\Gamma$-equivariant structure. This structure is induced from the $\Gamma$-equivariant structure on $\mathbb{P E}$ given for any $\gamma \in$ $\Gamma=\operatorname{Pic}^{0}(C)[n]$ by tensoring with the corresponding line bundle $L_{\gamma}$ and thinking of $\mathbb{P} \mathbf{E}$ as parametrizing lines in $\mathbf{E}$. Pulling back liftings along the map $\mathbb{P E} \rightarrow \mathbb{P E}$ induced by $\gamma$ defines the required action on $\alpha_{L}$.

Any $\gamma \in \Gamma$ induces a $\Gamma$-equivariant automorphism of $\alpha_{L \mid \mathrm{M}_{n}^{L, \gamma}}$, that is, a $\Gamma$-equivariant $\mu_{n^{-}}$ torsor $T_{L, \gamma}$ on $\mathrm{M}_{n}^{L, \gamma}$. The following proposition generalizes and relies on [HT03, Proposition 8.1].

Proposition A.1. The $\mu_{n}$-torsor $T_{L, \gamma}$ is trivial, and the $\Gamma$-equivariant structure is given by the character $\varrho_{\gamma}^{-q}$, where $q$ is the multiplicative inverse of $d$ modulo $n$.

Proof. Let $(E, \theta) \in \mathrm{M}_{n}^{L, \gamma}$. Since $(n, d)=1$, the Higgs bundle $(E, \theta)$ is stable, and therefore the automorphism induced by $E \otimes L_{\gamma} \cong E$ is given by a scalar. This implies that $\gamma$ acts trivially on $\mathbb{P} \mathbf{E}_{\mid \mathrm{M}_{n}^{L, \gamma}}$ and thus preserves any lifting of $\mathbb{P} \mathbf{E}$ over $\mathrm{M}_{n}^{L, \gamma}$. Hence, $T_{L, \gamma}$ is trivial.

By [HT03, Proposition 8.1], the $\Gamma$-equivariant structure on $T_{L, \gamma}$ is given by the character $\varrho_{\gamma}^{-q}$ in the case when $\gamma$ has maximal order $n$ (the extra assumption that $n$ is prime is only used later in the section of [HT03]).

For a general $\gamma \in \Gamma$, let $m=\operatorname{ord}(\gamma)$ and $r=n / m$. Since $\Gamma \cong(\mathbb{Z} / n \mathbb{Z})^{2 g}$, there exists a $\tilde{\gamma}$ of order $n$ such that $\gamma=\tilde{\gamma}^{r}$, and we have $\mathrm{M}_{n}^{L, \tilde{\gamma}} \subset \mathrm{M}_{n}^{L, \gamma}$. Since $T_{L, \gamma}$ is trivial, its $\Gamma$-equivariant structure is determined by its restriction to $\mathrm{M}_{n}^{L, \tilde{\gamma}}$, where by [HT03, Proposition 8.1], it is given by $\left(\varrho_{\tilde{\gamma}}^{-q}\right)^{r}=\varrho_{\gamma}^{-q}$.

\section{ACKNOWLEDGEMENTS}

We thank warmly Joseph Ayoub for his keenness in answering on the spot our naive questions on étale motives and Tamas Hausel for pointing out a mistake in an earlier version. The second author is grateful to Michael Groechenig and Paul Ziegler, as he learned many of the ideas used here from the collaborations with them. We are also grateful to the referee for a careful reading of the paper and many remarks and comments that were very helpful in the preparation of the final version.

\section{REFERENCES}

AEH15 G. Ancona, S. Enright-Ward and A. Huber, On the motive of a commutative algebraic group, Doc. Math. 20 (2015), 807-858.

AHP16 G. Ancona, A. Huber and S. Pepin Lehalleur, On the relative motive of a commutative group scheme, Algebr. Geom. 3 (2016), no. 2, 150-178; doi:10.14231/AG-2016-008.

Ayo07a J. Ayoub, Les six opérations de Grothendieck et le formalisme des cycles évanescents dans le monde motivique. I, Astérisque, 314 (2007).

Ayo07b_L_L Les six opérations de Grothendieck et le formalisme des cycles évanescents dans le monde motivique. II, Astérisque, 315 (2007). 


\section{MotiviC INTEGRATion ON THE Hitchin FibRATion}

Ayo14a A guide to (étale) motivic sheaves, Proceedings ICM Seoul 2014, Vol. II (Kyung Moon Sa, Seoul, 2014), 1101-1124.

Ayo14b_ La réalisation étale et les opérations de Grothendieck, Ann. Sci. Éc. Norm. Supér. (4) 47 (2014), no. 1, 1-145; doi:10.24033/asens.2210.

Bat98 V.V. Batyrev, Stringy Hodge numbers of varieties with Gorenstein canonical singularities, Integrable Systems and Algebraic Geometry (Kobe/Kyoto, 1997) (World Sci. Publ., River Edge, NJ, 1998), 1-32.

Bat99 Non-Archimedean integrals and stringy Euler numbers of log-terminal pairs, J. Eur. Math. Soc. (JEMS) 1 (1999), no. 1, 5-33, doi:10.1007/PL00011158.

BD96 V. V. Batyrev and D. I. Dais, Strong McKay correspondence, string-theoretic Hodge numbers and mirror symmetry, Topology 35 (1996), no. 4, 901-929; doi:10.1016/0040-9383(95)00051-8.

BLR90 S. Bosch, W. Lütkebohmert and M. Raynaud, Néron models, Ergeb. Math. Grenzgeb. (3), vol. 21 (Springer-Verlag, Berlin, 1990); doi:10.1007/978-3-642-51438-8.

BNR89 A. Beauville, M.S. Narasimhan and S. Ramanan, Spectral curves and the generalised theta divisor, J. reine angew. Math. 398 (1989), 169-179; doi:10.1515/crll.1989.398.169.

Bon09 M. V. Bondarko, Differential graded motives: weight complex, weight filtrations and spectral sequences for realizations; Voevodsky versus Hanamura, J. Inst. Math. Jussieu 8 (2009), no. 1, 39-97; doi:10.1017/S147474800800011X.

CH20 R. Cluckers and I. Halupczok, Evaluation of motivic functions, non-nullity, and integrability in fibers, 2020, arXiv:2004.09981.

CHL11 R. Cluckers, T. Hales and F. Loeser, Transfer principle for the fundamental lemma, in On the Stabilization of the Trace Formula (eds L. Clozel, M. Harris, J.-P. Labesse and B. C. Ngô) (Int. Press, Somerville, MA, 2011), 309-347.

CL08 R. Cluckers and F. Loeser, Constructible motivic functions and motivic integration, Invent. Math. 173 (2008), no. 1, 23-121; doi:10.1007/s00222-008-0114-1.

CL15_ Motivic integration in all residue field characteristics for Henselian discretely valued fields of characteristic zero, J. reine angew. Math. 701 (2015), 1-31; doi:10.1515/crelle2013-0025.

Del71 P. Deligne, Théorie de Hodge. II, Publ. Math. Inst. Hautes Études Sci. (1971), no. 40, 5-57; doi:10.1007/BF02684692.

DL98 J. Denef and F. Loeser, Motivic Igusa zeta functions, J. Algebraic Geom. 7 (1998), no. 3, $505-537$.

DL02 Motivic integration, quotient singularities and the McKay correspondence, Compos. Math. 131 (2002), no. 3, 267-290; doi:10.1023/A:1015565912485.

$\mathrm{DRR}^{+} 72$ P. Deligne, M. Raynaud, D. S. Rim, A. Grothendieck and M. Raynaud, Séminaire de Géométrie algébrique du Bois-Marie 1966-196\%. Groupes de monodromie en géométrie algébrique. (SGA 7 I), Lecture Notes in Math., vol. 228 (Springer-Verlag, Berlin, 1972); doi:10.1007/ $\mathrm{BFb0068688.}$

GH13 O. García-Prada and J. Heinloth, The y-genus of the moduli space of $\mathrm{PGL}_{n}$-Higgs bundles on a curve (for degree coprime to n), Duke Math. J. 162 (2013), no. 14, 2731-2749; doi: 10.1215/00127094-2381369.

GWZ20 M. Groechenig, D. Wyss and P. Ziegler, Mirror symmetry for moduli spaces of Higgs bundles via p-adic integration, Invent. Math. 221 (220), no. 2, 505-596; doi:10.1007/s00222-02000957-8.

HR15 T. Hausel and F. Rodriguez Villegas, Cohomology of large semiprojective hyperkähler varieties, Astérisque 370 (2015), 113-156.

HT03 T. Hausel and M. Thaddeus, Mirror symmetry, Langlands duality, and the Hitchin system, Invent. Math. 153 (2003), no. 1, 197-229; doi:10.1007/s00222-003-0286-7. 


\section{F. LOESER AND D. WYSS}

Hub00 A. Huber, Realization of Voevodsky's motives, J. Algebraic Geom. 9 (2000), no. 4, 755-799.

IS13 F. Ivorra and J. Sebag, Nearby motives and motivic nearby cycles, Selecta Math. (N.S.) 19 (2013), no. 4, 879-902; doi:10.1007/s00029-012-0111-5.

Lev98 M. Levine, Mixed motives, Math. Surveys Monogr., vol. 57, (Amer. Math. Soc., Providence, RI, 1998); doi:10.1090/surv/057.

LS03 F. Loeser and J. Sebag, Motivic integration on smooth rigid varieties and invariants of degenerations, Duke Math. J. 119 (2003), no. 2, 315-344; doi:10.1215/S0012-7094-03-11924-9.

Ngô10 B. C. Ngô, Le lemme fondamental pour les algèbres de Lie, Publ. Math. Inst. Hautes Études Sci. (2010), no. 111, 1-169; doi:10.1007/s10240-010-0026-7.

Ogg62 A. P. Ogg, Cohomology of abelian varieties over function fields, Ann. of Math. 76 (1962), no. 2, 185-212; doi:10.2307/1970272.

Yas04 T. Yasuda, Twisted jets, motivic measures and orbifold cohomology, Compos. Math. 140 (2004), no. 2, 396-422; doi:10.1112/S0010437X03000368.

Yas06 _ Motivic integration over Deligne-Mumford stacks, Adv. Math. 207 (2006), no. 2, 707761; doi:10.1016/j.aim.2006.01.004.

François Loeser francois.loeser@imj-prg.fr

Sorbonne Université, Institut de Mathématiques de Jussieu-Paris Rive Gauche, CNRS. Univ Paris Diderot, Campus Pierre et Marie Curie, case 247, 4 place Jussieu, 75252 Paris cedex 5, France

Dimitri Wyss dimitri.wyss@epfl.ch

EPFL/SB/ARG, Station 8, CH-1015 Lausanne, Switzerland 